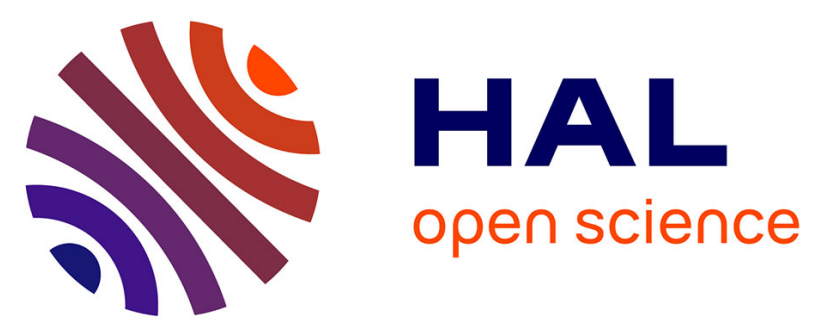

\title{
The emptying of a bottle as a test case for assessing interfacial momentum exchange models for Euler-Euler simulations of multi-scale gas-liquid flows
}

\author{
Samuel Mer, Olivier Praud, Hervé Neau, Nicolas Merigoux, Jacques \\ Magnaudet, Véronique Roig
}

\section{To cite this version:}

Samuel Mer, Olivier Praud, Hervé Neau, Nicolas Merigoux, Jacques Magnaudet, et al.. The emptying of a bottle as a test case for assessing interfacial momentum exchange models for Euler-Euler simulations of multi-scale gas-liquid flows. International Journal of Multiphase Flow, 2018, vol. 106, pp. 109-124. 10.1016/j.ijmultiphaseflow.2018.05.002 . hal-01801495

\section{HAL Id: hal-01801495 \\ https://hal.science/hal-01801495}

Submitted on 28 May 2018

HAL is a multi-disciplinary open access archive for the deposit and dissemination of scientific research documents, whether they are published or not. The documents may come from teaching and research institutions in France or abroad, or from public or private research centers.
L'archive ouverte pluridisciplinaire $\mathbf{H A L}$, est destinée au dépôt et à la diffusion de documents scientifiques de niveau recherche, publiés ou non, émanant des établissements d'enseignement et de recherche français ou étrangers, des laboratoires publics ou privés. 


\section{Open Archive TOULOUSE Archive Ouverte (OATAO)}

OATAO is an open access repository that collects the work of Toulouse researchers and makes it freely available over the web where possible.

This is an author-deposited version published in : http://oatao.univ-toulouse.fr/ Eprints ID : 20029

To link to this article : DOI:10.1016/j.ijmultiphaseflow.2018.05.002 URL : https://doi.org/10.1016/j.ijmultiphaseflow.2018.05.002

To cite this version : Mer, Samuel $\leftrightarrows$ and Praud, Olivier Hervé Véronique $\rightleftharpoons$ The emptying of a bottle as a test case for assessing interfacial momentum exchange models for Euler-Euler simulations of multi-scale gas-liquid flows. (2018) International Journal of Multiphase Flow, vol. 106. pp. 109-124. ISSN 0301-9322

Any correspondence concerning this service should be sent to the repository administrator: staff-oatao@,listes-diff.inp-toulouse.fr 


\title{
The emptying of a bottle as a test case for assessing interfacial momentum exchange models for Euler-Euler simulations of multi-scale gas-liquid flows
}

\author{
Samuel Mer ${ }^{\mathrm{a}}$, Olivier Praud ${ }^{\mathrm{a}}$, Herve Neau ${ }^{\mathrm{a}}$, Nicolas Merigoux $^{\mathrm{b}}$, Jacques Magnaudet ${ }^{\mathrm{a}}$, \\ Veronique Roig ${ }^{\mathrm{a}}$
}

a Institut de Mécanique des Fluides de Toulouse, IMFT, Université de Toulouse - CNRS, Toulouse, France

${ }^{\mathrm{b}}$ Electricité de France RED, 6 quai Wattier, Chatou 78401, France

Keywords:

Euler-Euler formulation

Bubbly flow

Multi-scale flow

\begin{abstract}
A B S T R A C T
Simulating gas-liquid flows involving a wide range of spatial and temporal scales and multiple topological changes remains a major challenge nowadays, as the computational cost associated with direct numerical simulation still makes this approach unaffordable. A common alternative is the two-fluid Euler-Euler formulation that avoids solving all scales at the price of semi-empirical closures of mass, momentum and energy exchanges between the two fluids. Many of such closures are available but their performances in complex flows are still in debate. Closures considering separately large gas structures and smaller bubbles and making these two populations evolve and possibly exchange mass according to their interactions with the surrounding liquid have recently been proposed. In order to assess the validity of some of these closures, we carry out an original experiment in a simple configuration exhibiting a rich succession of hydrodynamic events, namely the emptying of a water bottle. We simulate this experiment with the NEPTUNE_CFD code, using three different closure approaches aimed at modelling interfacial momentum exchanges with various degrees of complexity. Based on experimental results, we perform a detailed analysis of global and local flow characteristics predicted by each approach to unveil its potentialities and shortcomings. Although all of them are found to predict correctly the overall features of the emptying process, striking differences are observed regarding the distribution of the dispersed phase and its consequences in terms of liquid entrainment.
\end{abstract}

\section{Introduction}

Gas-liquid flows involving a broad range of bubble sizes are ubiquitous in geophysical and engineering configurations and applications, such as magmatic chimneys, submarine explosions, bubble columns or nuclear safety, to mention just a few. In such situations, the gas phase frequently involves a wide range of spatial and temporal scales, from large gas pockets to small dispersed bubbles. Moreover, dramatically different flow regimes may be encountered, characterized by distinct interaction mechanisms between the gas phase and the carrying liquid. Simulating such flows remains a major challenge nowadays, although massive efforts have been devoted during the last two decades to develop modelling strategies aimed at computing multiphase flows (Prosperetti and Tryggvason, 2007).
These strategies differ according to the level of accuracy they target and the computational resources they require. A first class of numerical techniques based on Direct Numerical Simulation (DNS) of the Navier-Stokes equations shares the same main challenge consisting in precisely localizing interfaces in the flow domain and imposing the proper jump conditions across them. Three main approaches have been proposed to track interfaces, namely the Volume Of Fluid (Hirt and Nichols, 1981), Level Set (Osher and Sethian, 1988) and Front Tracking (Unverdi and Tryggvason, 1992) methods (see also Scardovelli and Zaleski, 1999; Sethian and Smereka, 2003; Tryggvason et al., 2001 for reviews). Since then, these techniques have become mature and are now widely used to get insight into detailed mechanisms governing flow configurations with increasing complexity. For bubbly flows, this may range from those involving a single bubble rising at large Reynolds number (Cano-Lozano et al., 2016) to dispersed bubbly flows with up to $\mathcal{O}\left(10^{3}\right)$ bubbles moving at moderate Reynolds number (Bunner and Tryggvason, 2002). 
Despite the potentialities offered by these DNS approaches and their improvements associated with local grid refinement techniques (Popinet, 2009), their computational cost still makes them unable to simulate complex configurations, especially those involving multiple coalescence and break-up sequences. Such complex two-phase flows are usually computed using the much cruder Euler-Euler approach based on the so-called two-fluid model (Ishii, 1975; Ishii and Hibiki, 2006). In this framework, the governing equations are obtained after volume-averaging (or more formally ensemble-averaging) the local budgets, so that unknown terms occur at interfaces. As in any averaging scheme, closures are required to express these terms with respect to the primitive variables and their gradients. The accuracy of the simulations then depends tremendously on the validity of these closures. Many of them have been proposed for each flow configuration, e.g. separated flows, dispersed bubbly or particulate flows, etc. (see Drew and Passman, 1999 and Balachandar and Eaton, 2010 for reviews). In bubbly flows for instance, assuming non-deformable and mono-disperse bubbles, momentum interfacial exchange is usually modelled by considering drag, added-mass and shear-induced lift forces acting on individual bubbles, supplemented with turbulent diffusiophoresis and lubrification effects when the carrying flow is turbulent and walls are present, respectively. Applications of the twofluid approach to such flows, possibly with phase change, may be found for instance in Mimouni et al. (2010, 2011a, 2011b). Similarly, specific closures with various degrees of sophistication have been developed to simulate 'slug' flow configurations (Issa and Kempf, 2003; Issa et al., 2006) and separated nearly-horizontal flows (Vallée et al., 2008).

The key limitation of the above closures is that they are specific to the configuration for which they were calibrated and are unable to properly model the interfacial exchange mechanisms at work in another type of flow. This limitation can only be overcome if the modelling approach is made able to recognize which configuration is present at a given position in space and time. Two main streams of approaches were developed during the last two decades to reach this goal. The first of them consists in switching locally from DNS (based on either the Volume Of Fluid or the Level Set approach) to the two-fluid formulation wherever interfaces exhibit a characteristic size of the order of the grid cell (Černe et al., 2001; Tomiyama et al., 2006; Yan and Che, 2010). This technique was successfully employed to compute several gas-liquid flows dominated by fragmentation, e.g. a two-phase vortex or the unstable Rayleigh-Taylor configuration. The second approach consists in extending the two-fluid model to an arbitrary number of 'fields' or 'phases', each of them corresponding to a specific flow configuration or class of two-phase entities (e.g. small bubbles, large bubbles, slugs, etc). Occurrence of each of these configurations at a given time and position has to be identified in order to evaluate the corresponding volume fraction. As each 'phase' has its own velocity field, momentum closures have to be formulated to properly account for the interaction between two of them. This approach has for instance been applied to the upward bubbly pipe flow with several widely distinct bubble sizes and possible mass exchange between them, due to phase change (Krepper et al., 2008).

Although the above methodology was initially designed to deal with dispersed flows, it may be applied to separated flows as well, provided one is able to $(i)$ properly define a criterion allowing the occurrence of the 'separated' configuration to be detected, and (ii) derive realistic closure laws for the various separated flow regimes according to the interface roughness. This is the essence of the Large Interface Model (LIM) designed by Henriques (2006) and Coste (2013), as well as that of the Algebraic Interfacial Area Density (AIAD) model promoted by Höhne and Vallée (2010) and Deendarlianto et al. (2011). Mixed configurations in which separated and dispersed regions coexist within the flow may also be tackled within the framework of the $n$-field approach, provided the above criterion allows 'Large Interfaces' (hereinafter abbreviated as LI) corresponding to the separated configuration to be disentangled from small-scale interfaces, and distinct closure laws are employed for the dispersed and separated regions. This idea yielded several different modelling approaches, such as the Generalized Two-Phase Flow model (GENTOP, Hansch et al., 2012) or the Generalized Large Interface Model (GLIM, Merigoux et al., 2016). Examples of application of this type of approach to a gas jet impinging a free surface and a bubble column with bubbles bursting at the free surface may be found in the first reference.

Still in the context of the two-fluid and $n$-field formulations, several attempts were recently carried out to achieve a more realistic and accurate treatment of LI by taking explicitly into account surface tension effects (Bartosiewicz et al., 2008; Štrubelj et al., 2009; Gada et al., 2017). A technical difficulty arises in this type of approach, due to the natural tendency for numerical diffusion to spread stiff volume fraction gradients. Sharpening techniques have been proposed to counteract this effect and maintain well-defined separated 'phases', so that the LI may remain properly defined over time. A cutoff length must also be defined, so that interfaces with a characteristic size smaller than this critical length are no longer resolved and interactions between the corresponding dispersed phase and the continuous one are entirely modelled with the help of empirical closure laws. Last, an exchange procedure combining numerical requirements and basic physical principles has to be designed to allow a LI to break up into smaller bubbles, and such bubbles to coalesce and generate a LI. Such an approach has been implemented both in the aforementioned GENTOP formulation (Montoya et al., 2015), and in the NEPTUNE_CFD code where it is termed the Large Bubble Model (LBM, Denèfle et al., 2015; Mimouni et al., 2017). Preliminary assessment of this approach in canonical configurations, such as the KelvinHelmholtz and Rayleigh-Taylor instabilities, was reported by Fleau et al. $(2015,2016)$.

The aim of the present paper is to assess the validity of the above LIM, GLIM and LBM approaches implemented in the NEPTUNE_CFD software, by considering an academic but already significantly complex flow configuration and performing a one-toone comparison between original experiments carried out in that flow and computations making use of the above three models. The selected two-phase configuration, namely the emptying of a water bottle, is especially relevant for checking such modelling approaches, as it exhibits a wide range of temporal and spatial scales. Large air bubbles with diameters of the order of the bottle neck are periodically generated and rise within the bottle until they burst at the free surface below the top of the bottle. While ascending, these large bubbles undergo successive break-up events, yielding swarms of smaller bubbles, part of which may coalesce again and participate into the regeneration and reconfiguration of the large bubble population.

Few computational studies have been performed so far on this flow configuration. The most noticeable is that of Geiger et al. (2012) who simulated it with the help of the OpenFoam software in the framework of a Volume Of Fluid approach. They mainly focused on the influence of geometrical parameters and bottle inclination on the emptying time. However they assumed the liquid and air phases to be both isothermal and incompressible. As we shall see later, the latter assumption is highly questionable.

The present paper is organized as follows. Section 2 introduces the multi-field formulation and details the various approaches employed to model interfacial momentum exchanges in the NEPTUNE_CFD code. The experimental and computational configurations are described in Section 3. Section 4 discusses typical results obtained through both approaches on some quantities characteriz- 
ing the overall dynamics of the system. Section 5 focuses on local characteristics of the two-phase flow and examines the influence of the aforementioned models on the evolution and statistical distribution of these characteristics. Finally Section 6 summarizes the main findings of the study and draws some prospects for future work.

\section{2. n-field Approach and interfacial exchange models in NEPTUNE_CFD}

All simulations presented in this paper were performed with the release 4.0 of the NEPTUNE_CFD code jointly developed by EDF, CEA, IRSN and Framatome for more than a decade. The governing equations considered in this code are based on an extension to $n$ fields of the two-fluid model (Ishii and Hibiki, 2006). This technique is particularly well-suited for dispersed flows involving a single size of particles, drops or bubbles. In this context, the description reduces to two fields, namely a continuous carrying liquid phase and a dispersed one. In general, mass, momentum and energy conservation equations are solved for each field, with the assumption that they share the same pressure field. From a numerical viewpoint, all governing equations considered in NEPTUNE_CFD are discretized using a finite volume technique with collocated variables. The grid is unstructured and involves arbitrary shaped cells. A second order linear upwind scheme is used to update the volume fraction of each field. The velocity field is advanced thanks to a fractional step technique while the pressure field is computed with the help of the SIMPLE algorithm (Patankar and Spalding, 1972). An iterative coupling between energy and mass balances is used to enforce the simultaneous conservation of both quantities (Mimouni et al., 2008).

\subsection{Primary equations of the $\mathrm{n}$-field model}

We assume an isothermal flow, so that only the mass and momentum conservation equations need to be considered. Velocities and volume fractions, together with fluid properties (density and viscosity) are defined for each field $k$ at every point of the domain. The volume fractions, $\alpha_{k}$, satisfy

$\sum_{k} \alpha_{k}=1$

For each field $k$, the mass balance is written as

$\frac{\partial}{\partial t}\left(\alpha_{k} \rho_{k}\right)+\nabla \cdot\left(\alpha_{k} \rho_{k} \mathbf{u}_{k}\right)=\sum_{p \neq k} \Gamma_{p \rightarrow k}$,

where $\rho_{k}$ and $\mathbf{u}_{k}$ are the density and velocity of field $k$, and $\Gamma_{p \rightarrow k}$ denotes the mass exchange rate between fields $p$ and $k$. For any $p$ and $k$, this mass exchange rate must satisfy

$\Gamma_{p \rightarrow k}+\Gamma_{k \rightarrow p}=0$.

In two-fluid gas-liquid configurations, Eq. (3) merely expresses the interfacial mass balance, so that $\Gamma_{p \rightarrow k}$ is the interfacial mass exchange rate possibly due to phase change. No such phase change is considered in the simulations to be discussed later. However, when several distinct fields are employed to represent entities of different sizes within the same physical phase (e.g. large and small bubbles within the gas phase), coalescence and break-up events make the mass of each of these fields vary, so that $\Gamma_{p \rightarrow k}$ is generally nonzero.

The momentum balance for phase $k$ is written as

$$
\begin{aligned}
\frac{\partial}{\partial t}\left(\alpha_{k} \rho_{k} \mathbf{u}_{k}\right) & +\nabla \cdot\left(\alpha_{k} \rho_{k} \mathbf{u}_{k} \otimes \mathbf{u}_{k}\right)=-\alpha_{k} \nabla P+\alpha_{k} \rho_{k} \mathbf{g} \\
& +\nabla \cdot\left(\alpha_{k} \mu_{k}\left(\nabla \mathbf{u}_{k}+{ }^{\mathrm{T}} \nabla \mathbf{u}_{k}\right)\right)+\sum_{p \neq k} \mathbf{I}_{p \rightarrow k},
\end{aligned}
$$

where $\mu_{k}$ is the viscosity of field $k$ and $\mathbf{I}_{p \rightarrow k}$ represents the momentum exchange rate between fields $p$ and $k$. The latter may be split in the form

$\mathbf{I}_{p \rightarrow k}=\mathbf{I}_{p \rightarrow k}^{H}+\Gamma_{p \rightarrow k} \mathbf{u}_{p k}^{I}$,

where $\mathbf{u}_{p k}^{I}$ is the velocity at the interface between phases $p$ and $k$, and the first term in the right-hand side, $\mathbf{I}_{p \rightarrow k}^{H}$, represents the momentum exchange due to hydrodynamic forces, while the second is merely the momentum exchange associated with the mass exchange rate between the two phases. The interfacial momentum exchange term has to be modelled to close the set of equations.

If an interfacial tension, $\sigma_{p k}$, may be defined between phases $p$ and $k$, the corresponding interfacial momentum balance implies

$\mathbf{I}_{p \rightarrow k}+\mathbf{I}_{k \rightarrow p}=\lim _{V_{\Omega} \rightarrow 0} \frac{1}{V_{\Omega}} \int_{\Omega} \sigma_{p k} \kappa_{p k} \mathbf{n}_{p k} d A^{I}$,

where $\mathbf{n}_{p k}$ is the unit normal to the $p-k$ interface, $\kappa_{p k}=-\nabla \cdot \mathbf{n}_{p k}$ is the corresponding mean curvature, $d A^{I}$ is the elementary interfacial area and $\Omega$ denotes the control volume (which in computational practice corresponds to the grid cell), the volume of which is $V_{\Omega}$. In what follows, capillary effects are neglected in the LIM and GLIM approaches. We shall specify in due course how and under which conditions they are taken into account in the LBM. Note that, provided the interfacial velocity $\mathbf{u}_{p k}^{I}$ is continuous across the interface, the left-hand side of Eq. (6) reduces to $\mathbf{I}_{p \rightarrow k}^{H}+\mathbf{I}_{k \rightarrow p}^{H}$, owing to Eq. (3).

In the next four subsections, we detail the various closures and detection criteria used to express the interfacial momentum exchange in the three aforementioned models implemented in NEPTUNE_CFD. A summary of the characteristics and closure laws involved in each of these models is provided in Table 1.

\subsection{The dispersed bubbly flow model}

In a bubbly flow with mono-dispersed bubbles, the above set of equations reduces to the usual two-fluid formulation. The corresponding two fields are referred to as the continuous liquid $(\mathrm{cl})$ and the dispersed gas $(\mathrm{dg})$ phases, respectively. In such a flow, provided wall effects and turbulent dispersion are absent or have negligible effects, the interfacial momentum exchange is assumed to result from the sum of three independent contributions, namely a viscous drag force, $\mathbf{F}^{D}$, an added mass force, $\mathbf{F}^{A M}$, and a shearinduced lift force, $\mathbf{F}^{L}$ (Mimouni et al., 2011b).

The momentum transfer resulting from viscous drag is written as

$\mathbf{F}_{c l \rightarrow d g}^{D}=\frac{1}{8} A^{I} \rho_{l} C_{D}\left\|\mathbf{u}_{d g}-\mathbf{u}_{c l}\right\|\left(\mathbf{u}_{c l}-\mathbf{u}_{d g}\right)$,

where $A^{I}=\lim _{V_{\Omega} \rightarrow 0} \frac{1}{V_{\Omega}} \int_{\Omega} d S^{I}$ denotes the rate of interfacial area per unit volume which, for a mono-dispersed bubble distribution, may be expressed as a function of the volume fraction of the dispersed phase through the well-known relation $A^{I}=6 \alpha_{d g} / d_{d g}, d_{d g}$ denoting the bubble diameter. The drag coefficient $C_{D}$ depends on the bubble Reynolds number, $R e_{d g}=\rho_{l}\left\|\mathbf{u}_{d g}-\mathbf{u}_{c l}\right\| d_{d g} / \mu_{l}$, on the Bond number, $B o_{d g}=\left(\rho_{l}-\rho_{g}\right) g d_{d g}^{2} / \sigma$ ( $\sigma$ being the surface tension of the liquid), and on the volume fraction, $\alpha_{d g}$. NEPTUNE_CFD makes use of the empirical correlations established by Ishii and Zuber (1979) to express $C_{D}$ as a function of $R e_{d g}, B o_{d g}$ and $\alpha_{d g}$ in the various regimes encountered with rising bubbles, possibly in dense configurations.

The contribution of added mass effects to $\mathbf{I}_{p \rightarrow k}^{H}$ is written in the form (Zuber, 1964)

$\mathbf{F}_{c l \rightarrow d g}^{A M}=C_{A M} \frac{1+2 \alpha_{d g}}{1-\alpha_{d g}} \alpha_{d g} \rho_{l}\left(\frac{D \mathbf{u}_{c l}}{D t}-\frac{d \mathbf{u}_{d g}}{d t}\right.$, 
Table 1

Summary of the three closure models for the momentum interfacial exchange rate, $\mathbf{I}^{H}$.

\begin{tabular}{|c|c|c|c|c|c|}
\hline Model & $\begin{array}{l}\text { Number } \\
\text { of gas fields }\end{array}$ & $\begin{array}{c}\text { Cutoff } \\
\text { length } \mathrm{cg} / \mathrm{dg}\end{array}$ & $\begin{array}{c}\text { Diameter of } \\
\text { dispersed bubbles }\end{array}$ & $\begin{array}{l}\text { Forces acting on } \\
\text { LI }\end{array}$ & $\begin{array}{l}\text { Forces acting on } \\
\text { dispersed bubbles }\end{array}$ \\
\hline LIM & 1 & - & - & $\begin{aligned} \mathbf{I}_{c l \rightarrow c g}^{H} & =\mathbf{F}_{c l \rightarrow c g}^{F}(\text { Eq. }(10)) \\
& +\mathbf{F}_{c l \rightarrow c g}^{P}(\text { Eq. (11)) }\end{aligned}$ & $\mathbf{I}_{c l \rightarrow d g}^{H}=\mathbf{F}_{c l \rightarrow c g}^{P}($ Eq. (11)) \\
\hline GLIM & 1 & $l_{c} \approx \Delta$ & $d_{g}$ & $\begin{aligned} & \mathbf{I}_{c l \rightarrow c g}^{H}= \mathbf{F}_{c l \rightarrow c g}^{F} \\
&+\mathbf{F}_{c l \rightarrow c g}^{P}\end{aligned}$ & $\begin{aligned} \mathbf{I}_{c l \rightarrow d g}^{H} & =\mathbf{F}_{c l \rightarrow d g}^{D}(\text { Eq. (7)) } \\
& +\mathbf{F}_{c l \rightarrow d g}^{A M} \text { (Eq. (8)) } \\
& +\mathbf{F}_{c l \rightarrow d g}^{L} \text { (Eq. (9)) }\end{aligned}$ \\
\hline LBM & 2 & $l_{c}=8 \Delta$ & $d_{g}$ & $\begin{aligned} \mathbf{I}_{c l \rightarrow c g}^{H} & =\mathbf{F}_{c l \rightarrow c g}^{\sigma}(\text { Eq. }(10)) \\
& +\mathbf{F}_{c l \rightarrow c g}^{D}(\text { Eqs. (15)-(17)) }\end{aligned}$ & $\begin{aligned} \mathbf{I}_{c l \rightarrow d g}^{H} & =\mathbf{F}_{c l \rightarrow d g}^{D}(\text { Eq. (7)) } \\
& +\mathbf{F}_{c l \rightarrow d g}^{A M}(\text { Eq. (8)) } \\
& +\mathbf{F}_{c l \rightarrow d g}^{L}(\text { Eq. (9)) }\end{aligned}$ \\
\hline
\end{tabular}

where $C_{A M}=0.5$ is the added mass coefficient of an isolated sphere, $\frac{D \mathbf{u}_{c l}}{D t}=\frac{\partial \mathbf{u}_{c l}}{\partial t}+\mathbf{u}_{c l} . \nabla \mathbf{u}_{c l}$ and $\frac{d \mathbf{u}_{d g}}{d t}=\frac{\partial \mathbf{u}_{d g}}{\partial t}+\mathbf{u}_{d g} . \nabla \mathbf{u}_{d g}$ are the local accelerations of the liquid and dispersed phases, respectively. These two distinct expressions of the accelerations are consistent with the theoretical expressions known to hold in an inviscid fluid (Auton et al., 1988). In the limit of small-but-finite volume fractions, the weighting by $\alpha_{d g}$ reduces to $\alpha_{d g}\left(1+3 \alpha_{d g}\right)$ which is also consistent with theoretical results (Biesheuvel and Spoelstra, 1989).

Last, the momentum transfer resulting from shear-induced lift effects is assumed to take the form

$\mathbf{F}_{c l \rightarrow d g}^{L}=C_{L} \alpha_{d g} \rho_{l}\left(\mathbf{u}_{c l}-\mathbf{u}_{d g}\right) \times \omega_{c l}$,

where $\omega_{c l}=\nabla \times \mathbf{u}_{c l}$ is the local vorticity in the liquid phase. When the Bond number is small, the lift coefficient $C_{L}$ is set to 0.5 which is the theoretical value corresponding to a sphere translating in a uniform inviscid shear flow (Auton, 1987), a result also known to hold for spherical bubbles moving in a viscous fluid provided $R e_{d g} \gtrsim 10^{2}$ (Legendre and Magnaudet, 1998). When the Bond number is of $\mathcal{O}(1)$, deformation alters the magnitude of the shearinduced lift force and may even reverse it (Adoua et al., 2009). In this regime, the empirical correlation $C_{L}=\mathcal{F}\left(B o_{d g}, R e_{d g}\right)$ established by Tomiyama et al. (2002) in a simple shear flow is applied.

\subsection{The Large Interface Model}

The $n$-field formulation may be adapted to the description of separated (or 'stratified') two-phase flows, for instance to deal with situations in which a high-speed gas shears a liquid layer. Two of the main attempts in that direction are the Algebraic Interfacial Area Density (AIAD) model implemented in the ANSYS CFX code (Höhne and Vallée, 2010; Deendarlianto et al., 2011) and the Large Interface Model (LIM) developed by Coste (2013) within the NEPTUNE_CFD code. The LIM comprises two main ingredients, namely a recognition algorithm aimed at detecting 'large' interfaces (LI) and a specific closure for the interfacial momentum exchange rate.

\subsubsection{Detection of a large interface}

In this model, a LI is captured thanks to a three-cell stencil (so-called LI3C algorithm): one cell contains the interface, with a mixture of the two phases (characterized by a non-zero value of the product $\alpha_{c l} \alpha_{c g}$ ), whereas one cell on each side of the interface is only filled with one phase. The former cell is identified based on the magnitude of the liquid volume fraction gradient, $\left\|\nabla \alpha_{c l}\right\|$, which for a separated configuration is identical to the rate of interfacial area per unit volume, $A^{I}$. The detection is achieved by comparing each component of $\nabla \alpha_{c l}$ to a prescribed threshold value. A cell is said to contain a $\mathrm{LI}$ if at least one of these components exceeds the threshold. Then, the neighbouring liquid and gas cells are identified by moving away from that cell in the direction normal to the interface, characterized by the unit vector $\mathbf{n}_{c l}=\nabla \alpha_{c l}|| \nabla \alpha_{c l} \|^{-1}$. Knowing the components of $\mathbf{n}_{c l}$ and the distribution of $\alpha_{c l}$ allows the position and orientation of the interface within the interfacecontaining cell to be determined. With this information at hand, distances between the interface and the neighbouring collocation points for the gas and liquid velocities may be evaluated. The tangential components of these neighbouring velocities are also obtained by projecting them onto the interface plane, making it possible to evaluate the gradient of these tangential components in the direction normal to the interface. Then the shear stress on both sides of the interface is estimated by applying a near-wall treatment qualitatively similar to that routinely employed to determine the so-called friction velocity in a turbulent boundary layer over a rigid wall. Here, continuity of shear stresses across the LI implies that the friction velocities in the two phases, $u_{c g}^{*}$ and $u_{c l}^{*}$, satisfy $\rho_{g} u_{c g}^{* 2}=\rho_{l} u_{c l}^{* 2}$. Coste (2013) devised a complex procedure aimed at taking into account the influence of the possible subgrid-scale roughness of the LI on $u_{c g}^{*}$ and $u_{c l}^{*}$. For this, he referred to the state diagram established by Brocchini and Peregrine (2001a,b) to distinguish between smooth, wavy and 'knobbly' interfaces. More detail on the detection algorithm and determination of the friction velocity may be found in the original reference (Coste, 2013).

\subsubsection{Momentum exchange across a large interface}

Once a cell is recognized to contain a LI and the friction velocities are determined, the tangential component of the interfacial momentum exchange rate within it is assumed to result from the frictional force density

$\mathbf{F}_{c l \rightarrow c g}^{F}=\rho_{g} u_{c g}^{* 2} A^{I} \frac{\left(\mathbf{u}_{c l}-\mathbf{u}_{c g}\right)}{\left\|\mathbf{u}_{c l}-\mathbf{u}_{c g}\right\|}$.

An additional contribution to $\mathbf{I}_{c l \rightarrow c g}^{H}$ is added in the normal direction for a purely numerical purpose. The role of this term is to enforce the equality of the normal components of $\mathbf{u}_{c l}$ and $\mathbf{u}_{c g}$ on a LI. Indeed, these two velocity fields being treated as independent variables and no interfacial tension acting at $\mathrm{cl}-\mathrm{cg}$ interfaces, there is in general no chance that this equality is achieved 'naturally'. Consequently it is enforced by introducing a penalization force density, $\mathbf{F}_{c l \rightarrow c g}^{P}$, in the form

$\mathbf{F}_{c l \rightarrow c g}^{P}=\alpha_{c l} \alpha_{c g}\left(\alpha_{c g} \rho_{g}+\alpha_{c l} \rho_{l}\right) \frac{C_{\tau}}{\Delta t}\left[\left(\mathbf{u}_{c l}-\mathbf{u}_{c g}\right) \cdot \mathbf{n}_{c l}\right] \mathbf{n}_{c l}$,

where $\Delta t$ is the numerical time step, $C_{\tau}$ is an empirical coefficient, and the weighting factor $\alpha_{c l} \alpha_{c g}$ ensures that $\mathbf{F}_{c l \rightarrow c g}^{P}$ is nonzero only in cells containing a LI. Thanks to the above definition of $\mathbf{F}_{c l \rightarrow c g}^{P}$, any difference between the normal components of $\mathbf{u}_{c l}$ and $\mathbf{u}_{c g}$ on a LI results in a large magnitude of this artificial force if $\Delta t$ is small. Hence, selecting a large value of $C_{\tau} / \Delta t$ guarantees that the difference between the two normal velocity components remains negligibly small in cells containing a LI.

The LIM approach summarized above was specifically developed to simulate separated two-phase flows. This model does not apply to a dispersed configuration. Indeed, although no LI is 

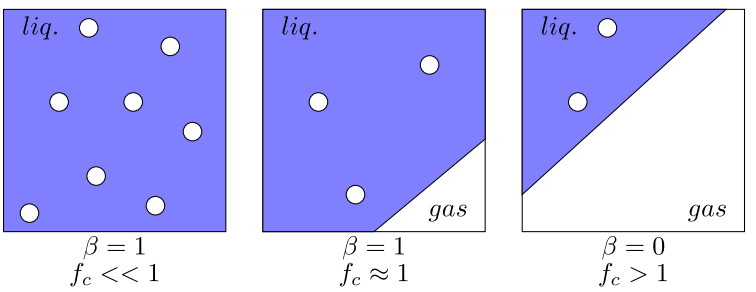

Fig. 1. Schematic representation of the role of $f_{c}$ and $\beta$ in the weighting procedure of the momentum closure defining the GLIM approach. The square represents the unit grid cell $\Omega$.

present in that case (as rightly identified by the detection algorithm), most cells exhibit non-zero values of the product $\alpha_{c l} \alpha_{c g}$. Thus, if the LIM approach is selected, the penalization force density defined in Eq. (11) is applied to these cells, providing a misrepresentation of the momentum exchange between the two phases.

\subsection{The Generalized Large Interface Model}

The idea underlying this model is to combine the capabilities of those described in Sections 2.2 and 2.3, so as to simulate multiscale flow configurations in which LI and dispersed bubbles coexist within the flow domain.

To this end, the interfacial momentum transfer closure has to be adapted to the local flow configuration. The first step is to detect LI throughout the domain by using the algorithm described in Section 2.3.1. Similar to the LIM approach, the momentum exchange closure $\mathbf{I}_{c l \rightarrow c g}^{H}=\mathbf{F}_{c l \rightarrow c g}^{F}+\mathbf{F}_{c l \rightarrow c g}^{P}$ is applied to cells containing a LI. Then, in cells that do not contain a LI but have a non-zero gas volume fraction, the dispersed bubbly flow model of Section 2.2 with an interfacial momentum exchange rate $\mathbf{I}_{c l \rightarrow d g}^{H}=\mathbf{F}_{c l \rightarrow d g}^{D}+\mathbf{F}_{c l \rightarrow d g}^{A M}+\mathbf{F}_{c l \rightarrow d g}^{L}$ is applied. To avoid superimposing both models in cells where $\alpha_{c l} \alpha_{c g} \neq 0$, the total momentum exchange rate within such cells is defined in a composite manner as $\gamma \mathbf{I}_{c l \rightarrow d g}^{H}+(1-\gamma) \mathbf{I}_{c l \rightarrow c g}^{H}$. The weighting pre-factor, $\gamma$, is selected in the form (Merigoux et al., 2016)

$\gamma=\beta\left(1-\operatorname{Min}\left(f_{c}, 1\right)\right)$ with $f_{c}=6 \frac{V_{\Omega}}{S_{\Omega}} \mathbf{n}_{\Omega} \cdot \nabla \alpha_{c l}$,

where $S_{\Omega}$ denotes the area of the outer surface that bounds the control volume $\Omega$, and $\mathbf{n}_{\Omega}$ is the outer unit normal to that surface. In Eq. (12), $\beta$ is a pre-factor that smoothly varies from 0 to $1 \mathrm{ac}-$ cording to the local value of the liquid volume fraction, $\alpha_{c l}$. More specifically, $\beta$ is set to 0 if $\alpha_{c l}<0.5$, which is considered to correspond to locations involving a LI. Conversely, $\beta=1$ if $\alpha_{c l}>0.7$, where the flow structure is considered to be dominated by the presence of dispersed bubbles. Last, $\beta$ follows the linear variation $\beta=\left(\alpha_{c l}-0.5\right) / 0.2$ if $0.5 \leq \alpha_{c l} \leq 0.7$. However, a fraction of a LI may still be present in cells with $\alpha_{c l}>0.5$. In such cells, it is obviously desirable to lower the influence of the dispersed phase and increase that of the LI. The correction function $f_{c}$ helps satisfying this request by lowering $\gamma$ if the rate of interfacial area associated with the LI present in the cell (which is approximately $\left.\left\|\nabla \alpha_{c l}\right\|\right)$ is of the same order as the surface-to-volume ratio of the grid cell, $S_{\Omega} / V_{\Omega}$ (see Fig. 1).

It is important to realize that in this model, the 'dispersed' ( $d g$ ) and 'continuous' ( $\mathrm{cg}$ ) gas phases actually refer to the same field. It is only the weighting factor, $\gamma$, that selects whether the local flow structure is close to a separated or a dispersed configuration. This selection criterion allows the 'generation' of a dispersed or continuous gas phase only in a limited number of configurations, mostly driven by grid resolution and numerical limitations. More specifically, starting from a configuration in which all interfaces are properly resolved, the GLIM approach may later detect

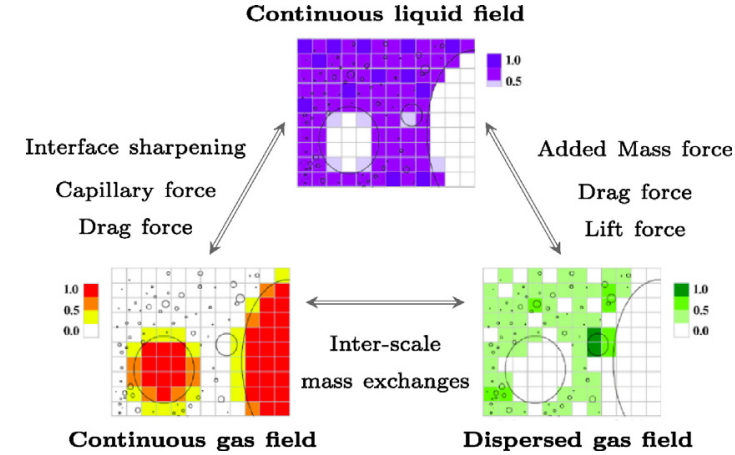

Fig. 2. Schematic of the inter-phase couplings involved in the Large Bubble Model (adapted from Denèfle et al., 2015).

a dispersed phase either if two LI get very close to one another (which also happens if the curvature of an interface becomes locally very large), or if the interfacial region containing a LI gradually thickens, owing to numerical diffusion. Conversely, starting from a purely dispersed gas-liquid configuration, a LI may only be created if the flow conditions or boundary conditions force the gradient of the gas volume fraction to reach locally a large value (e.g. a plume of rising bubbles hitting a horizontal wall). Last, it must be kept in mind that the dispersed phase is assumed to be monodispersed and that the ingredients brought to the GLIM approach by the models described in Sections 2.2 and 2.3 do not allow the bubble diameter, $d_{d g}$, to be predicted. Instead this diameter must be prescribed as a function of the average cell size, $\Delta_{a}$. In the simulations to be described below, this ratio is set to $d_{d g} / \Delta_{a} \approx 0.4$ (see Section 3.2).

\subsection{The Large Bubble Model}

The LBM (Denèfle, 2013; Denèfle et al., 2015; Mimouni et al., 2017 ) is the most sophisticated of the models considered in the present study. Here, distinct dispersed $(d g)$ and continuous ( $c g$ ) gas phases are considered, making the LBM a 3-field model involving separate $\mathrm{cl} \rightarrow \mathrm{cg}$ and $\mathrm{cl} \rightarrow d g$ momentum exchange closures. The $\mathrm{cl} \rightarrow d g$ closure is achieved thanks to the force density expressions detailed in Section 2.2, whereas the large gas structures are considered as a continuous phase separated from the carrying liquid by LI that are fully resolved and experience a capillary force.

Compared to LIM and GLIM, the extra modelling effort in the LBM rests essentially in the treatment of the LI which, in addition to the computation of the capillary force, involves an interface sharpening procedure and a specific closure for the interfacial drag force coupling the $\mathrm{cl}$ and $\mathrm{cg}$ fields. Furthermore, mass exchange terms associated with possible coalescence and fragmentation need to be implemented in the $d g$ and $c g$ fields. Fig. 2, adapted from Denèfle et al. (2015), summarizes the phase coupling procedures involved in the LBM approach.

\subsubsection{Computation of the capillary force and interface sharpening procedure}

The capillary force that takes place at these LI is evaluated thanks to the Continuum Surface Force (CSF) approach devised by Brackbill et al. (1992), in which the interfacial surface force density, i.e. the right-hand side of Eq. (6), is transformed into a volume force density, $\mathbf{F}_{k}^{\sigma}$. For phase $k=c l$ or $c g$, the latter is written as

$\mathbf{F}_{k}^{\sigma}=\alpha_{k} \sigma \kappa_{k p} \nabla \alpha_{k}$ with $p=c g, c l$,

where the interface mean curvature, $\kappa_{k p}=-\nabla \cdot \mathbf{n}_{k p}$, is computed using the unit normal pointing toward phase $k$, i.e. $\mathbf{n}_{k p}=$ $\nabla \alpha_{k}|| \nabla \alpha_{k} \|^{-1}$. 
Defining and computing the mean curvature, say $\kappa_{l g}$, at a liquid-gas interface makes sense only if this interface may be properly identified and described on the computational grid. A well-known issue encountered when attempting to track sharp interfaces using a purely hyperbolic transport equation for the volume fraction is the smearing of the discontinuity (Rudman, 1997; 1998; Sato and Ničeno, 2012). A common approach to limit this effect consists in introducing an additional viscous HamiltonJacobi equation ensuring an artificial compression of the interface (Sethian, 1999). NEPTUNE_CFD follows this path by solving the additional equation (Olsson and Kreiss, 2005; Olsson et al., 2007)

$\frac{\partial \alpha_{k}}{\partial \tau}+\nabla \cdot \alpha_{k}\left(1-\alpha_{k}\right) \mathbf{n}_{k p}=\epsilon \nabla^{2} \alpha_{k}, \quad k, p=c l, c g$.

The pseudo-time, $\tau$, and the pseudo-viscosity, $\epsilon$, are directly connected with the local cell size, $\Delta$. Following Štrubelj et al. (2009), values $\tau=\frac{\Delta}{32}$ and $\epsilon=\frac{\Delta}{2}$ are respectively selected. With this choice, the final thickness of the interfacial layer within which $\alpha_{k}$ varies from 0 to 1 is $\delta_{\alpha}=5 \Delta$ whatever the initial spreading of the $\alpha_{k}$ distribution. Eq. (14) is solved in conservative form in order to improve mass conservation (Fleau, 2017).

\subsubsection{Drag force on large interfaces}

Since the capillary force is taken into account and a sharpening procedure is applied, an artificial penalization force like that of Eq. (11) is no longer needed in the LBM approach to guarantee negligibly small relative normal velocities on a LI. Moreover, extensive tests of the LBM carried out by Fleau (2017) using a frictional drag force model only taking into account the fluid densities yielded quite poor results in the case of large bubbles rising in low-viscosity liquids, suggesting that fluid viscosities must enter the interfacial drag law. Similar findings were noticed with the AIAD model in applications involving free surfaces, and led Höhne and Vallée (2010) and Porombka and Höhne (2015) to develop alternative closure laws for the interfacial drag law to be applied on such interfaces. That frictional drag laws independent of the fluid viscosities fail to properly mimic the momentum transfer at gas-liquid interfaces is no surprise since the flow in the liquid close to such an interface almost obeys a shear-free condition rather than a no-slip one (provided contamination by surfactants is negligible). An important consequence of this difference is that the drag force on uncontaminated bubbles grows linearly rather than quadratically with the rise velocity (Batchelor, 1967). In NEPTUNE_CFD, this state of affairs led Fleau (2017) (see also Mimouni et al., 2017) to assume that the interfacial momentum transfer toward a $c g$ entity involves a characteristic length scale $l_{g}$ and is proportional to $\mu_{l} l_{g}\left(\mathbf{u}_{c l}-\mathbf{u}_{c g}\right)$, so that the momentum exchange rate per unit volume may be written in the form

$\mathbf{F}_{c l \rightarrow c g}^{D b}=\alpha_{c g} \mu_{l} l_{g}^{-2}\left(\mathbf{u}_{c l}-\mathbf{u}_{c g}\right)$.

This closure is used as long as $\alpha_{c g} \leq 0.3$, which is usually considered as the upper limit of the dispersed bubbly flow regime under homogeneous conditions (Taitel et al., 1980). Application of a similar reasoning to the 'opposite' case of large drops moving within a gas in regions where $\alpha_{c g} \geq 0.7$ yields

$\mathbf{F}_{c l \rightarrow c g}^{D d}=\alpha_{c l} \mu_{g} l_{l}^{-2}\left(\mathbf{u}_{c l}-\mathbf{u}_{c g}\right)$,

where $l_{l}$ is the length scale characterizing the interfacial momentum exchange in that configuration. In the intermediate range $0.3<\alpha_{c g}<0.7$, the momentum exchange rate is assumed to vary linearly in between the above two laws, namely

$\mathbf{F}_{c l \rightarrow c g}^{D}=f_{b d} \mathbf{F}_{c l \rightarrow c g}^{D b}+\left(1-f_{b d}\right) \mathbf{F}_{c l \rightarrow c g}^{D d}$,

with $f_{b d}\left(\alpha_{c g}\right)=2.5 \times\left(0.7-\alpha_{c g}\right)$. It is worth pointing out that the closure provided by Eq. (16) is much more questionable than that of Eq. (15) since the gas flow almost obeys a no-slip condition at a liquid-gas interface if $\mu_{g} / \mu_{l} \ll 1$, suggesting that a quadratic drag law would physically be more appropriate in that case.

The length scales $l_{i}(i=g, l)$ involved in Eqs. (15)-(17) are considered to depend on the product $\alpha_{c l} \alpha_{c g}$ (which may range from 0 to 0.25 ) in such a way that they both tend toward $d_{d g} / 3 \sqrt{2}$ when $\alpha_{c l} \alpha_{c g} \rightarrow 0$ (the factor $3 \sqrt{2}$ ensures that Stokes' drag law is recovered in that limit), whereas they become proportional to $\alpha_{c i}|| \nabla \alpha_{c i} \|^{-1}$ for $\alpha_{c l} \alpha_{c g} \gtrsim 0.1$ and a linear variation is assumed for intermediate values of $\alpha_{c l} \alpha_{c g}$. Since $\left\|\nabla \alpha_{c i}\right\|^{-1}$ is proportional to $\Delta$ thanks to the interface sharpening procedure, this definition makes the characteristic length scales of the momentum exchange at LI depend on the cell size.

\subsubsection{Cutoff length scale and mass exchanges between the continuous and dispersed gas phases}

Numerical requirements associated with the computation of the mean curvature $\kappa_{l g}$ of $c l-c g$ interfaces determine the characteristic size of the smallest gas structures that can be fully resolved. This critical size, $l_{c}$, is taken as the cutoff length scale corresponding to the separation between the $d g$ and $c g$ gas phases. In NEPTUNE_CFD, the computation of $\kappa_{l g}$ involves a 5-cell stencil in each grid direction. It is then a simple matter to show that the stencils required to compute the curvature at the two opposite points of the diameter of a circular interface overlap if this diameter is less than $5 \sqrt{2} \approx 7.2$ grid cells (Denèfle et al., 2015). For this reason, the value $l_{c} / \Delta=8$ is selected. Noting that the corresponding critical mean curvature is $\kappa_{c}=2 / l_{c}=(4 \Delta)^{-1}$, one has $\kappa_{c} V_{\Omega}\left(\delta_{\alpha} \Delta\right)^{-1}=$ $1 / 20$. Hence the cutoff criterion may be generalized by requiring that the capillary force is applied only if $\kappa_{l g} V_{\Omega}|| \nabla \alpha_{k} \| \Delta^{-1}$ is less than $1 / 20(k=c l, c g)$. Interfaces satisfying this criterion are considered as $\mathrm{LI}$ and the corresponding gas structures belong to the $\mathrm{cg}$ phase. Conversely, neither the interface sharpening procedure nor the capillary force density $\mathbf{F}_{k}^{\sigma}$ is applied to interfaces exhibiting a mean curvature larger than $\kappa_{c}$.

The corresponding small gas entities are transferred to the $d g$ phase as described below (see Eq. (19)). Mass exchanges between the $c g$ and $d g$ fields arise due to coalescence and break-up events. In the present context, the definition of these events relies on numerical rather than physical criteria, since the distinction between the $c g$ and $d g$ fields depends entirely on the grid resolution. Numerical coalescence of $d g$ bubbles giving rise to a $c g$ gas structure is assumed to occur when (i) the local gas fraction exceeds the critical value $\alpha_{d g_{c}}=0.3$ (Taitel et al., 1980), and (ii) the norm of the local gradient of the gas volume fraction, $\left\|\nabla \alpha_{d g}\right\|$, exceeds a threshold value. As the interface sharpening procedure yields a typical volume fraction gradient $\left\|\nabla \alpha_{c l}\right\|=\left\|\nabla \alpha_{c g}\right\|=\delta_{\alpha}^{-1}$ within interfacial regions, this threshold is set to $\left(2 \delta_{\alpha}\right)^{-1}$. Based on these requirements, the rate at which the dispersed phase coalesces is expressed in the form

$\Gamma_{c g+}=\alpha_{c g} \alpha_{d g} \rho_{g} \frac{C_{+}}{\Delta t} H\left(\alpha_{d g}-\alpha_{d g_{c}}\right) H\left(2 \delta_{\alpha}\left\|\nabla \alpha_{d g}\right\|-1\right)$,

where $H$ denotes the Heaviside function and $C_{+}$is an $\mathcal{O}(1)$ constant. Similarly, the rate at which $c g$ gas entities turn into dispersed bubbles is assumed to be

$\Gamma_{c g_{-}}=\alpha_{c g} \alpha_{d g} \rho_{g} \frac{C_{-}}{\Delta t} H\left(\delta_{\alpha}\left\|\nabla \alpha_{k}\right\| \frac{\kappa_{l g}}{\kappa_{c}}-1\right)$,

where $C_{-}$is another $\mathcal{O}(1)$ constant. The net mass exchange rate from the dispersed gas field toward the $c g$ field is then

$\Gamma_{d g \rightarrow c g}=\Gamma_{c g+}-\Gamma_{c g-}$.

Note that the presence of the $\alpha_{c g} \alpha_{d g}$ pre-factor in $\Gamma_{c g+}$ and $\Gamma_{c g}$ ensures that mass exchanges may only take place at locations where both gas fields are present. The interaction of the dispersed gas phase with the carrying continuous liquid takes place through 


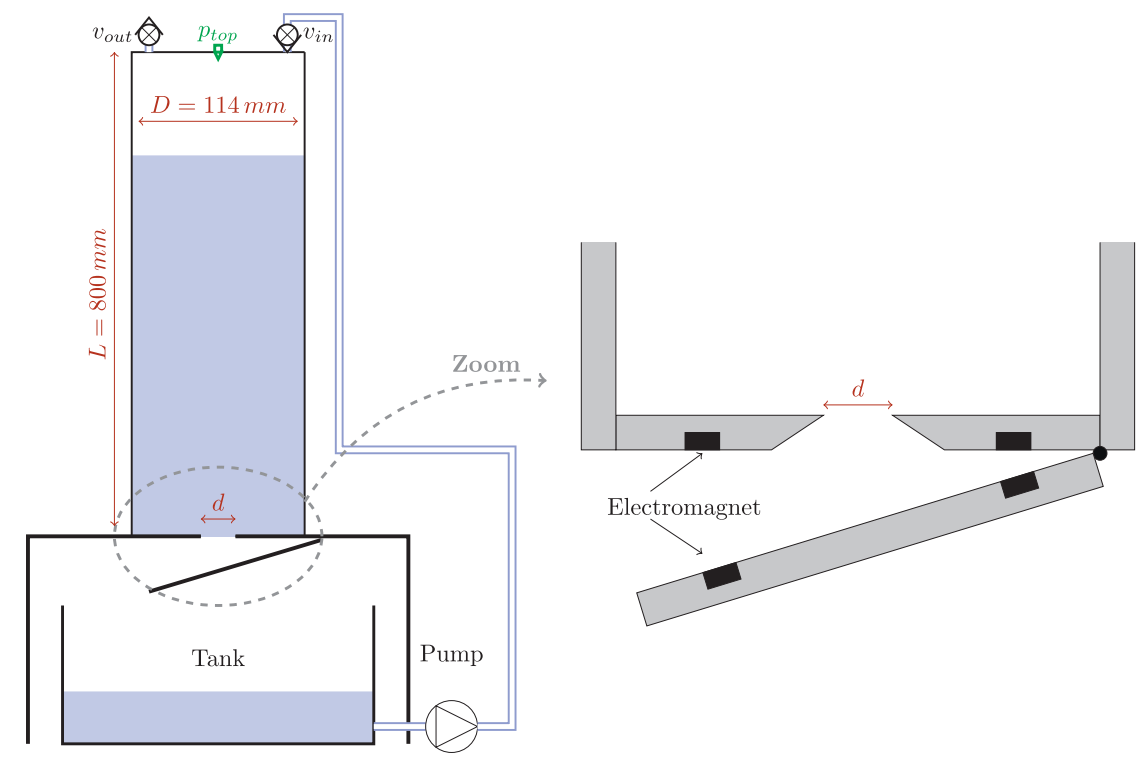

Fig. 3. Schematic of the experimental set-up.

the interfacial forces defined in Eqs. (7)-(9), assuming the $d g$ phase to be monodispersed and made of bubbles with diameter $d_{d g} \ll l_{c}$. Similar to the GLIM approach, $d_{d g}$ has to be specified as a fraction of $\Delta_{a}$ (see Section 3.2).

\section{Experimental and computational configurations}

\subsection{Experimental set-up}

The experimental set-up is sketched in Fig. 3. It consists of a Plexiglas $^{\mathrm{TM}}$ cylindrical vessel with diameter $D=114 \mathrm{~mm}$ and height $L=800 \mathrm{~mm}$. The top end of the cylinder is closed with a blank flange. Conversely, its bottom section is opened thanks to a central circular thin-walled hole with a diameter $d=35 \mathrm{~mm}$. This hole has a bevelled edge making a sharp $20^{\circ}$ angle with the cylinder base. The bottle neck is closed by a gate mounted on two hinges and equipped with five electromagnets ensuring a fast opening. Before a test, the gate is closed, the valves $v_{\text {in }}$ and $v_{\text {out }}$ are opened and the bottle is filled up to an altitude $z=z_{0}$ with tap water, using the circulation pump. Once filling is completed, the pump is turned off and the two valves are closed. As $v_{\text {out }}$ remains opened during the filling process, the initial pressure at the top of the bottle is the atmospheric pressure, $p_{a t m}$. The gate is quickly opened at time $t=0$. Water starts to flow out of the bottle in a succession of jets separated by the generation of large air bubbles at the neck. Then these bubbles rise within the water column and reach the free surface after a sequence of complex reconfigurations.

The air pressure at the centre of the top end of the bottle, $p_{t o p}$, is monitored with a pressure sensor (Keller, PR-23). Images of the emptying process are recorded with a CMOS camera (Photon Lines, PCO $1200 \mathrm{HS}$ ) at a speed of $400 \mathrm{fps}$. These images make it possible to follow the displacement of the upper free surface and give insight into the bubble dynamics. The camera and the pressure sensor are synchronized through a TTL signal that triggers the electromagnet, thus the gate opening.

\subsection{Computational set-up and conditions}

The computational geometry is sketched in Fig. 4. Similar to the experimental device, the 'numerical bottle' consists of a cylinder with diameter $D=114 \mathrm{~mm}$ and height $L=800 \mathrm{~mm}$. To relax grid

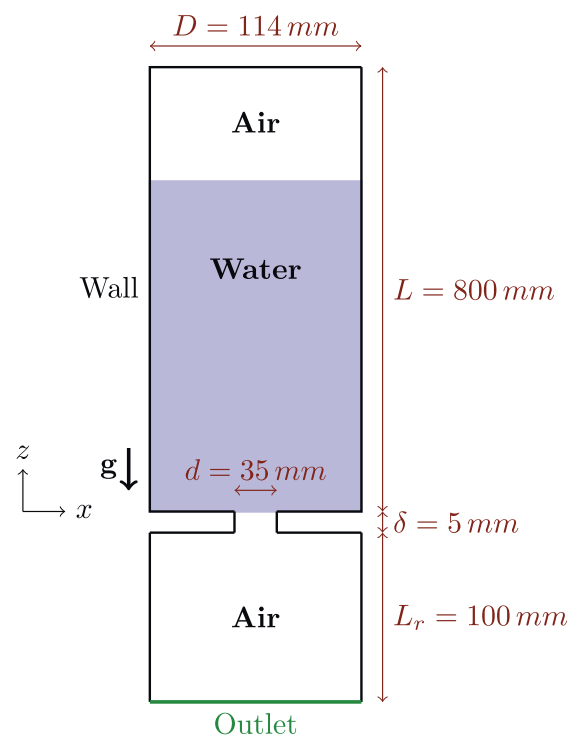

Fig. 4. Cross-sectional view of the computational domain; $\mathbf{g}=-g \mathbf{e}_{\mathbf{z}}$ denotes the gravity vector.

constraints, the bottle neck with diameter $d=35 \mathrm{~mm}$ is assumed to be a thin cylinder with height $\delta=5 \mathrm{~mm}$, thus slightly differing from the bevelled geometry used in the experiment. No-slip conditions are imposed on all walls and intersections between walls and iso- $\alpha_{l}$ surfaces take place at right angle, corresponding to a $90^{\circ}$ contact angle.

The outlet boundary condition plays a crucial role in the present configuration. To prevent the liquid outflow and bubble generation at the bottle neck from being disturbed by the outlet, the latter is moved away from the neck by adding a buffer region with length $L_{r}=0.1 \mathrm{~m}$ and diameter $D$ below the bottle neck, as depicted in Fig. 4.

The initial conditions refer to the experimental case with an initial water height $z_{0} / L=0.75$. Water and air are initially at rest and the initial pressure in both air regions is the atmospheric pressure. Water properties at $20^{\circ} \mathrm{C}$ are extracted from the CATHARE rou- 


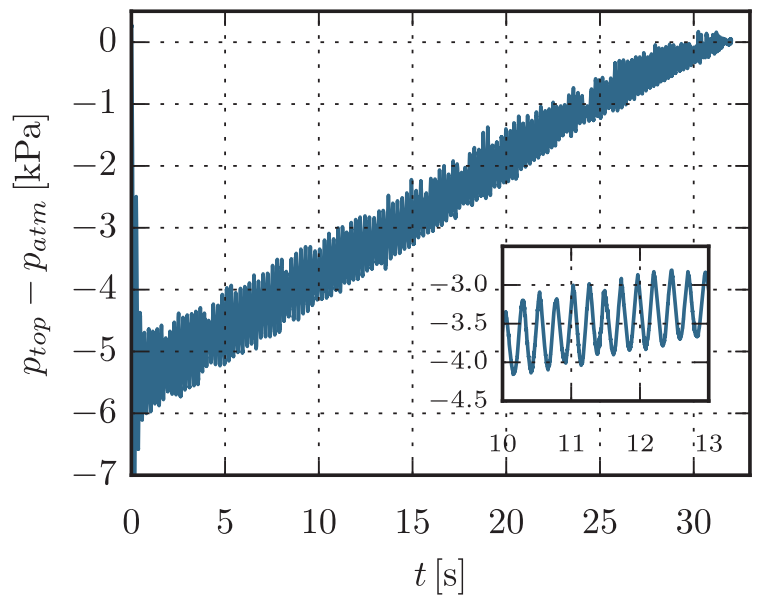

Fig. 5. Evolution of the pressure at the top of the bottle for an initial water height $z_{0} / L=0.75$. The inset shows a closer view at the oscillations in the range $10 \mathrm{~s} \leq t \leq 13 \mathrm{~s}$.

tines encapsulated in NEPTUNE_CFD, namely $\rho_{l}=997.24 \mathrm{~kg} \cdot \mathrm{m}^{-3}$, $\mu_{l}=1 \times 10^{-3}$ Pa.s and $\sigma=7.28 \times 10^{-2}$ N.m. Air viscosity is set to $\mu_{g}=1.8 \times 10^{-5}$ Pa.s. To assess the possible role of air compressibility, incompressible simulations as well as compressible ones (in which the propagation of density/pressure waves is fully resolved) are carried out. In incompressible cases, air density is set to $\rho_{a}=1.20 \mathrm{~kg} \cdot \mathrm{m}^{-3}$. In contrast, when compressibility is taken into account, air is assumed to behave as an isothermal ideal gas, the density of which obeys $\rho_{a}=p\left(R_{\text {air }} T\right)^{-1}$, where $p$ denotes the local pressure, $R_{\text {air }}=287.06 \mathrm{~J} \cdot \mathrm{kg}^{-1} \cdot \mathrm{K}^{-1}$ is the perfect gas constant, and the temperature $T$ is set to $293.15 \mathrm{~K}$. Under such isothermal conditions, the density derivative with respect to pressure is merely $\left(\partial \rho_{a} / \partial p\right)_{T}=\left(R_{\text {air }} T\right)^{-1}$

With the above physical properties, the capillary length of the fluid set-up is $l_{\sigma}=\left(\sigma / \rho_{l} g\right)^{1 / 2} \approx 2.7 \mathrm{~mm}$ ( $g$ denoting gravity). Hence $d / l_{\sigma} \approx 13$, which guarantees that capillary effects in the neck region have a negligible influence on the emptying dynamics.

The simulations are run over $5 \mathrm{~s}$, with a constant time step of $0.05 \mathrm{~ms}$. All simulations discussed below are carried out on a three-dimensional computational domain with approximately 2.1 millions cells, which yields an average grid size $\Delta_{a} \approx 1.6 \mathrm{~mm}$ corresponding to a dimensionless length ratio $\Delta_{a} / D \approx 0.014$. With this grid, the diameter $d_{d g}$ prescribed for the dispersed bubbles in both the GLIM and LBM approaches is set to $1 \mathrm{~mm}$. It yields a bubble volume about $15 \%$ of the averaged cell volume, $\Delta_{a}^{3}$, which is consistent with the assumption of the presence of a dispersed phase and allows us to properly resolve a significant part of the gas content. The main characteristics of the three closure models employed in the computations are summarized in Table 1. We stress that no turbulence model is used in any of these computations. The calculations are run in parallel mode on 100 processors of the EOS supercomputer from the CALMIP supercomputing mesocentre. Computations making use of the LIM, GLIM and LBM approaches consume approximately 7500,5200 and $11,500 \mathrm{~h}$ in total CPU time, respectively.

\section{Global results}

\subsection{Preliminary experimental observations}

The evolution of the pressure $p_{\text {top }}$ throughout the emptying process is shown in Fig. 5 in the case of an initial water height $z_{0} / L=0.75$

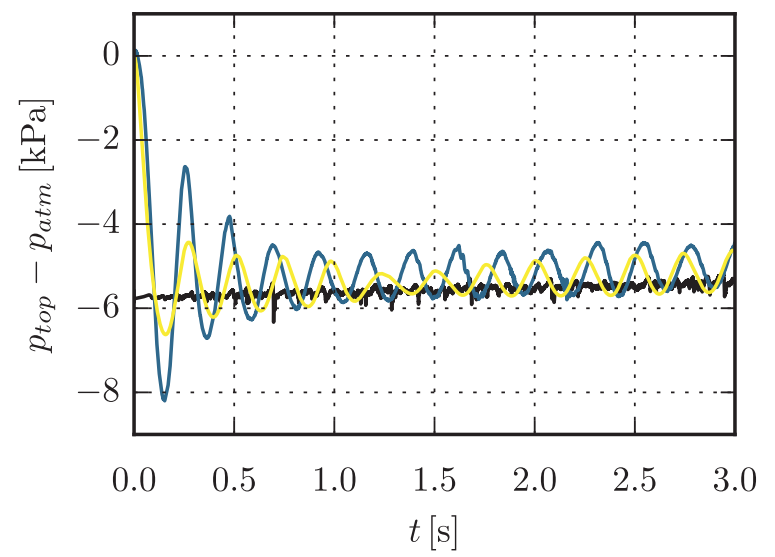

Fig. 6. Evolution of the pressure difference $p_{t o p}-p_{a t m}$ obtained in experiments (blue line), incompressible (black line) and compressible (yellow line) simulations with the LIM approach, respectively. (For interpretation of the references to colour in this figure legend, the reader is referred to the web version of this article.)

Just after the opening, the pressure immediately drops from $p_{a t m}$ to a lower value, $p_{a t m}-\Delta p^{\text {init }}$. This initial drop is merely due to the hydrostatic pressure corresponding to the initial height of the water column, so that $\Delta p^{i n i t} \approx \rho_{l} g z_{0}$. The pressure then rises almost linearly over time until it goes back to the atmospheric pressure, $p_{a t m}$, at the end of the emptying process.

A closer look at the pressure signal (see the inset in Fig. 5) reveals almost sinusoidal oscillations around a slowly increasing value, $p_{\text {top }}^{e q}(t)$, corresponding to the instantaneous hydrostatic equilibrium. These oscillations have a typical period $T_{o s} \approx 0.20 \mathrm{~s}$, much shorter than the emptying time. They find their root in the alternation of bubble generation and liquid ejection events, as described by Clanet and Searby (2004). The evolution of the equilibrium pressure, $p_{\text {top }}^{e q}$, obeys the relation $p_{\text {top }}^{e q}(t)=p_{a t m}-4 g M_{w}(t) /\left(\pi D^{2}\right)$, where $M_{w}(t)$ is the mass of water in the bottle at time $t$ (hence $4 M_{w}(t) /\left(\rho_{l} \pi D^{2}\right)$ is the instantaneous water height). In the present case, the linear evolution of $p_{t o p}^{e q}$ yields a constant mass flow rate $d M_{w} / d t \approx-0.19 \mathrm{~kg} . \mathrm{s}^{-1}$, corresponding to a cross-sectional averaged velocity $U_{a v} \approx 0.2 \mathrm{~m} . \mathrm{s}^{-1}$ at the bottle neck, which yields a Reynolds number based on the neck diameter of approximately 7000 .

\subsection{Influence of air compressibility}

A typical pressure signal recorded by the pressure sensor is displayed in Fig. 6, still with the initial condition $z_{0} / L=0.75$. Pressure oscillations start right after the gate opening and are present until the end of the emptying process. Based on their experiments, Clanet and Searby (2004) attributed these oscillations to the compressibility of the air buffer at the top of the bottle. However, an oscillatory behaviour was also observed in the incompressible simulations carried out by Geiger et al. (2012). To assess whether or not compressibility has to be taken into account to properly reproduce the emptying process, we carried out both compressible and incompressible simulations. The pressure signal corresponding to both sets of conditions is compared with the experimental signal in Fig. 6. Only computational results obtained with the LIM approach are displayed in this figure but the other two models reveal similar trends.

Large differences are observed with the two modelling assumptions. When air is considered incompressible, the computed pressure signal does not display the sinusoidal oscillations observed in experiments. It rather follows a noisy evolution characterized by small-amplitude high-frequency fluctuations. The magnitude of the initial drop and the subsequent linear increase of 

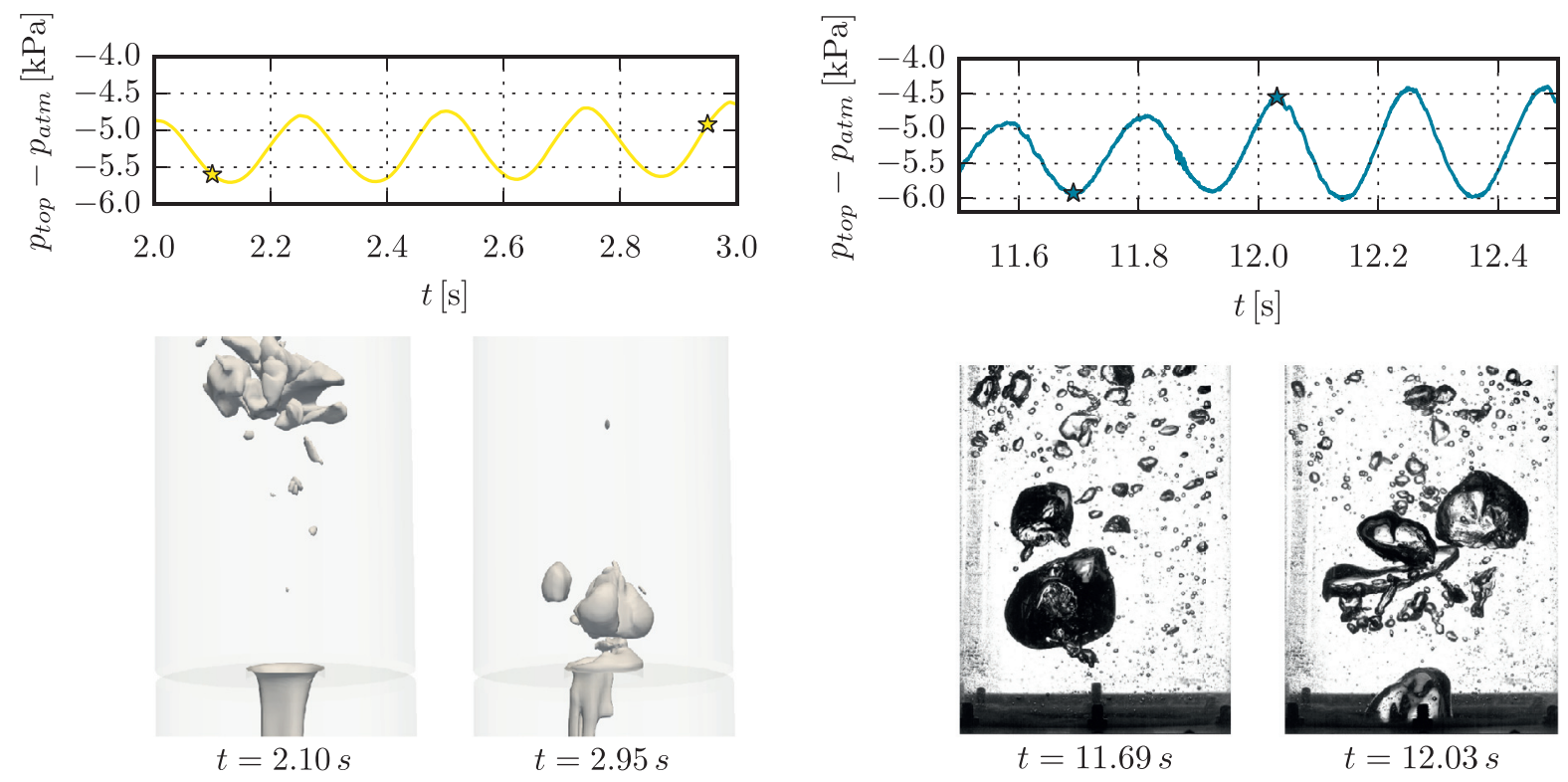

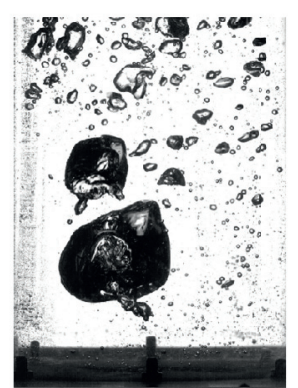

$t=11.69 \mathrm{~s}$

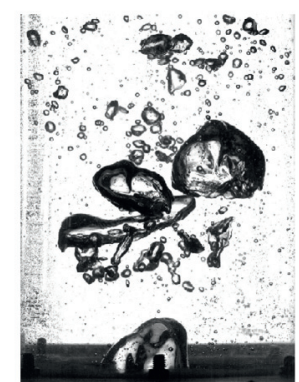

$t=12.03 \mathrm{~s}$

(a)

(b)

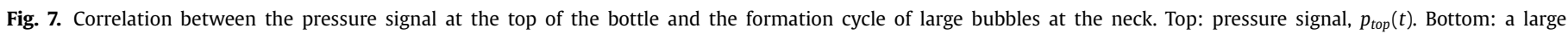

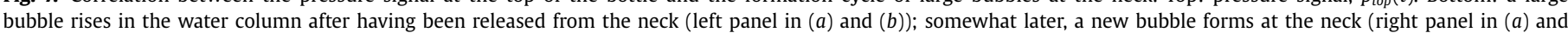

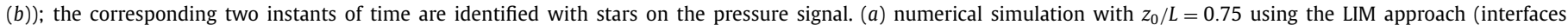
are identified using the iso-contour $\alpha_{c l}=0.5$ ); (b) experiment with $z_{0} / L=1$.

the equilibrium pressure are in qualitative agreement with the characteristics of the $p_{\text {top }}^{e q}(t)$ component of the experimental signal. However we shall see in Section 4.4 that there are significant quantitative differences with a direct consequence regarding the predicted time-averaged flow rate. In contrast, when air compressibility is taken into account, the pressure signal displays the expected oscillations, with an amplitude and a frequency in reasonable agreement with the experimental findings, except during the initial transient when the magnitude of the oscillations is clearly underestimated. From this comparison, it may be concluded that air compressibility is essential to correctly reproduce pressure oscillations. Consequently all numerical results discussed below were obtained through compressible simulations. The underestimate of the oscillation amplitude during the initial transient may have several origins. One of them could be that the evolution is not strictly isothermal, given the magnitude of the sudden pressure drop. There may also be some influence of the bevelled shape of the neck which is not considered in the computations.

To reveal the importance of air compressibility on the flow dynamics, the connection between the pressure signal at the top of the bottle and the life cycle of large bubbles generated at its neck is displayed in Fig. 7. This figure shows that the observed pressure oscillations are the direct signature of the water jets and large air bubbles successively generated at the bottle neck. When the liquid flows out (left panels in $(a)$ and $(b)$ ), the free surface moves downward, inducing an expansion of the air buffer at the top of the bottle, hence a drop in the local pressure. As $p_{\text {top }}(t)$ decreases below the hydrostatic equilibrium value $p_{t o p}^{e q}(t)$, an upward force starts acting on the water column, making the generation of a new bubble at the neck and its release at the bottom of the water column possible. The free surface then moves upward, making the pressure increase, which corresponds to the start of a new cycle (right panels in $(a)$ and $(b))$.

\subsection{Influence of the interfacial momentum exchange model on the pressure in the top air buffer}

Fig. 8 reveals the influence of the interfacial momentum exchange model (LIM, GLIM or LBM) on the evolution of the pressure recorded at the top of the bottle. As already noticed, a transient takes place during the first $1 \mathrm{~s}$ following the gate opening. This first stage is characterized by pressure oscillations, the amplitude of which decreases exponentially until it reaches an almost timeindependent value close to $1 \mathrm{KPa}$. All three models are found to severely underestimate the oscillation amplitude during that stage. While the initial pressure drop recorded in the experiment is approximately $8 \mathrm{kPa}$, its predicted amplitude barely exceeds $6 \mathrm{kPa}$. It is noteworthy that all three computational curves perfectly superimpose during the first three oscillations. This suggests that the initial transient is mainly governed by compressibility effects, with little influence of the detailed flow dynamics. Beyond this transient, say for $t>1.5 \mathrm{~s}$, the pressure oscillates around an equilibrium value. All models predict fairly well the oscillation amplitude as well as the equilibrium pressure during that second stage.

Experimental and computational determinations of the time period, $T_{o s}$, of these oscillations are compared in Fig. 9. The theoretical prediction derived by Clanet and Searby (2004) is also shown as reference. According to this prediction, the oscillation period depends on the gas thermodynamic characteristics and water height in the form $T_{o s}=L\left(\gamma_{p v} p_{\text {top }} / \rho_{g}\right)^{-1 / 2} \Phi(z(t) / L)$ with $\Phi(z) \approx$ $\left\{\frac{z}{L}\left(1-\frac{z}{L}\right)\right\}^{1 / 2}$, where $\gamma_{p v}$ is the adiabatic index, i.e. the ratio of the two specific heats of the gas, and $z(t)$ is the current height of the water in the bottle.

The experimental and computational periods are obtained through a sliding Fourier transform of the corresponding pressure signals, using a succession of $1 \mathrm{~s}$ time windows with a $50 \%$ overlap between two consecutive windows. In order to limit the influence of the finite-size window effect on the results, time windows are projected on a half cosine. The theoretical prediction of Clanet and Searby (2004) is found to be in general agreement with observa- 


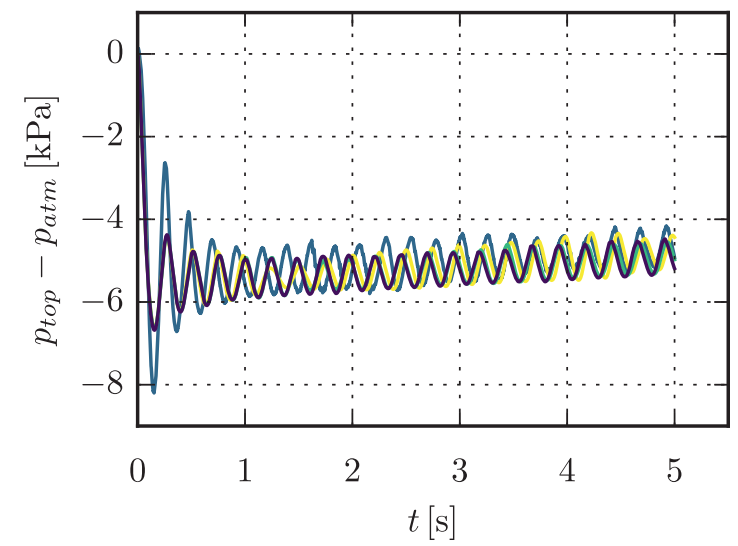

Fig. 8. Evolution of the pressure at the top of the bottle. The experimental signal (blue line) is compared with computational predictions obtained with the three different interfacial momentum exchange models (yellow: LIM, green: GLIM, purple: LBM). (For interpretation of the references to colour in this figure legend, the reader is referred to the web version of this article.)

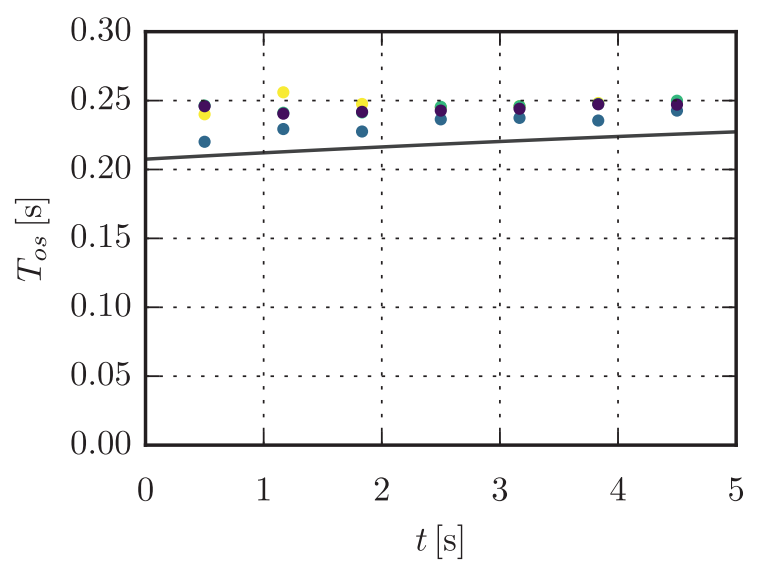

Fig. 9. Evolution of the pressure oscillation period throughout the emptying process. Predictions of the various interfacial momentum exchange models are compared with experimental data and theoretical predictions provided by the model of Clanet and Searby (2004) (black solid line). Symbols obey the colour code defined in the caption of Fig. 8.

tions. It slightly under-predicts the actual period but correctly captures its slow increase. All three interfacial momentum exchange models give similar predictions, although LBM is seen to be generally marginally more accurate. All models slightly overestimate the actual oscillation period, especially during the first half of the process. The discrepancy may be up to $10 \%$ and reduces as time goes on.

The above observations indicate that the pressure signal is barely sensitive to the details of the interfacial momentum exchange. All three models are found to provide reasonable predictions of the amplitude and time period of pressure oscillations, except during the initial transient.

\subsection{Influence of the closure model on the liquid flow rate}

The computed and experimentally determined average liquid flow rates are reported in Table 2. When air compressibility is not considered, the emptying process is significantly slowed down, with a mass flow rate underestimated by more than $35 \%$. This confirms that air compressibility plays an essential role in the overall flow dynamics and cannot be ignored in computations. Once compressibility is taken into account, the mean flow rate obtained with the LIM approach is seen to be in very good agreement with the experimental value, whereas the GLIM and LBM com- putations in which the dispersed bubble diameter is set to $1 \mathrm{~mm}$ under-predict it by $13 \%$. This difference may be anticipated from Fig. 8, where a slight downshift of the equilibrium pressure predicted by the GLIM and LBM formulations is noticed. Nevertheless, we regard the much closer agreement obtained with the LIM approach as mostly fortuitous, since the precise value of the predicted flow rate depends on the closures used in the interfacial momentum exchange model. This is easily understood by considering the budget of the total (i.e. kinetic plus potential) mechanical energy, $e_{M}=\alpha_{c l} \rho_{l}\left(\frac{1}{2} \mathbf{u}_{c l}^{2}+g z\right)$, of the liquid enclosed within the cylinder. From Eq. (4) it is straightforward to show that

$$
\begin{aligned}
\frac{d}{d t} & \int_{V_{c}} e_{M} d V+\int_{S_{n}} e_{M} \mathbf{u}_{c l} \cdot \mathbf{n}_{n} d S \\
= & \int_{S_{n}} \alpha_{c l}\left(-P \mathbf{n}_{n}+2 \mu_{l} \mathbb{S}_{c l} \cdot \mathbf{n}_{n}\right) d S \\
& -2 \mu_{l} \int_{V_{c}} \alpha_{c l} \mathbb{S}_{c l}: \mathbb{S}_{c l} d V+\int_{V_{c}} \sum_{p \neq c l} \mathbf{I}_{p \rightarrow c l} \cdot \mathbf{u}_{c l} d V,
\end{aligned}
$$

where $\mathbb{S}_{c l}=\frac{1}{2}\left(\nabla \mathbf{u}_{c l}+{ }^{\mathrm{T}} \nabla \mathbf{u}_{c l}\right), \mathbf{n}_{n}$ is the outer unit normal to the neck surface, $S_{n}$, and $V_{c}$ is the volume of the cylinder. The last term in the right-hand side of Eq. (21) is the rate of work resulting from the momentum exchange between the liquid and the gas phase $p(p=c l, c g)$ within the cylinder. Due to the presence of this term, it is clear that the rate at which the initial potential energy of the liquid is converted into kinetic energy depends directly on $\mathbf{I}_{p \rightarrow c l}$, hence on the closures discussed in Section 2. For instance, any change in the evaluation of the interfacial friction velocity, $u_{c g}^{*}$, involved on large interfaces in the LIM and GLIM approaches (see Eq. (10)), or in that of the length scales $l_{g}$ and $l_{l}$ involved in the LBM formulation (see Eqs. (15) and (16), respectively) modifies this balance, which directly impacts the liquid flow rate. A similar effect is expected to take place when the closure laws used to model the momentum exchange with the dispersed phase are modified. To illustrate this influence, we carried out two additional simulations based on the GLIM and LBM approaches, with $d_{d g}$ set to $2 \mathrm{~mm}$. The corresponding results are provided in the last two rows of Table 2. Compared to those obtained with $d_{d g}=1 \mathrm{~mm}$, the flow rate is found to be increased by $1 \%$ and $1.2 \%$, respectively.

This influence of the closure models on the flow rate makes us consider that the two most robust indications conveyed by the results displayed in Table 2 are that $(i)$ the LIM, GLIM and LBM formulations in which air compressibility is taken into account all provide a reasonable estimate of the mean flow rate, and that (ii) under present conditions, this prediction is lower by typically $10 \%$ with the latter two approaches, compared to that obtained with the LIM formulation. This lowering originates in the much more significant presence of a dispersed phase in the simulations performed with the former two models, as shown in Figs. 10 and 11 below. In both cases, the rise of dispersed bubbles induces an average upward motion in the liquid through the interfacial momentum exchange model. Thus, these small bubbles somewhat hamper the liquid outflow, which yields a slightly slower emptying dynamics. In other terms, a larger part of the initial potential energy of the liquid is transferred to interfaces when the GLIM or LBM formulation is used, reducing the part that can be converted into kinetic energy, hence the liquid flow rate. In Table 2, the effect of an increase of $d_{d g}$ to $2 \mathrm{~mm}$ is seen to be slightly stronger in the LBM simulation, which is no surprise since a larger part of the gas content is treated as dispersed in this formulation, owing to the larger cutoff length involved (see Table 1).

Having doubled $d_{d g}$ divides the number of bubbles in the dispersed phase by a factor of eight, as changing $d_{d g}$ leaves the volume fraction $\alpha_{d g}$ unchanged and only modifies the momentum transferred from the liquid to the dispersed bubbles. This is why the corresponding effect is small with both models. More signifi- 
Table 2

Influence of the selected model on the prediction of the time-averaged water flow rate. In the experiment, this quantity is determined by dividing the initial mass of liquid by the total emptying time, while in computations it is obtained by time-averaging the instantaneous liquid flow rate throughout the simulation.

\begin{tabular}{|c|c|c|c|c|}
\hline \multirow[t]{2}{*}{ Nature of the simulation } & \multirow[t]{2}{*}{ Momentum exchange model } & \multicolumn{3}{|c|}{$\begin{array}{l}\text { Emptying mass flow rate } \\
\qquad d M_{\text {liq }} / d t\left[{\left.\mathrm{~g} . \mathrm{s}^{-1}\right]}^{-1}\right.\end{array}$} \\
\hline & & Qnum & $Q_{e x p}$ & $\left(Q_{e x p}-Q_{n u m}\right) / Q_{e x p}[\%]$ \\
\hline incomp. & LIM & -123.0 & -191.2 & -35.5 \\
\hline comp. & LIM & -189.4 & -191.2 & -1.0 \\
\hline comp. & GLIM $-d_{d g}=1 \mathrm{~mm}$ & -166.4 & -191.2 & -13.0 \\
\hline comp. & $\mathrm{LBM}-d_{d g}=1 \mathrm{~mm}$ & -166.3 & -191.2 & -13.1 \\
\hline comp. & GLIM $-d_{d g}=2 \mathrm{~mm}$ & -168.0 & -191.2 & -12.2 \\
\hline comp. & $\mathrm{LBM}-d_{d g}=2 \mathrm{~mm}$ & -168.3 & -191.2 & -12.0 \\
\hline
\end{tabular}

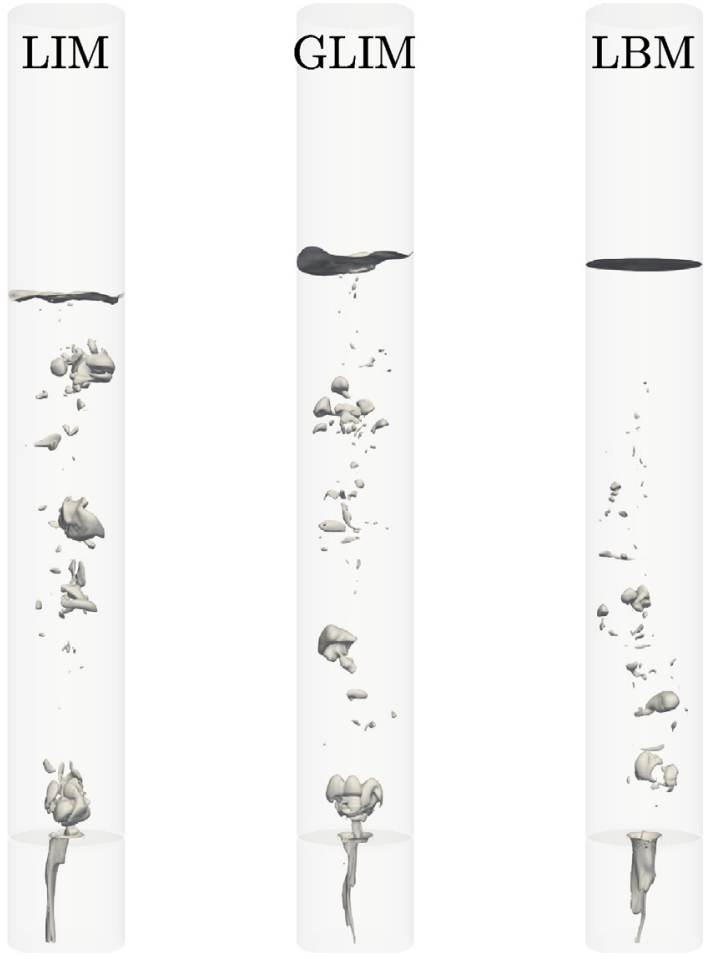

Fig. 10. Visualization of the large bubbles in the water column at $t=4.2 \mathrm{~s}$. Interfaces are identified with the iso-contour $\alpha_{c l}=0.5$.

cant variations of the flow rate related to the presence of the dispersed gas phase would presumably be observed if the cutoff criterion were tuned, either by modifying the way $\beta$ and $f_{c}$ are defined in Eq. (12) in the GLIM approach, or by changing the ratio $l_{c} / \Delta$ in the LBM representation. We did not attempt such tests up to now but plan to perform them in the future.

\section{Influence of the interfacial momentum exchange model on the local flow characteristics}

\subsection{Qualitative observations}

The three models mostly differ in the way large gas structures involving LI coexist with smaller bubbles and interact with the surrounding water flow. To obtain more insight into the potential differences between these models, examination of suitable local characteristics of the gas and liquid dynamics is required.

A snapshot of the distribution of the large gas bubbles within the bottle, as predicted by each model, is displayed in Fig. 10. With the LIM approach, the large bubbles formed at the neck rise toward the free surface with little size variation. This is no surprise since no momentum transfer mechanism toward the dispersed phase exists in that model. Actually, in the present configuration in which the initial interface reduces to a single LI, small bubbles can only be created due to the numerical smearing of the successive interfaces, which, as time proceeds, prevents some of them from being considered as LI by the detection algorithm (see Section 2.3.1). The observed behaviour is significantly different with the other two models. In both cases, the large bubbles generated at the bottle neck undergo substantial successive reconfigurations and their average size decreases as they rise, owing to fragmentation. With the LBM, the fragmentation rate is such that the continuous gas phase is virtually absent in the upper part of the water column, having been transferred earlier to the dispersed phase.

Fig. 11 is similar to Fig. 10 but the computational snapshots have been coloured according to the local volume fraction of the dispersed phase, so as to better reveal the complete structure of the multi-scale bubble swarm (a video based on the GLIM simulation is linked to the paper). The corresponding experimental snapshot (with $z_{0} / L=1$ as initial condition) is also shown, to serve as a reference. This experimental snapshot highlights the broad distribution of air bubble sizes within the bottle. Close to the bottom, air is mostly contained within a few large bubbles which are subject to fragmentation and gradually evolve in a swarm of much smaller bubbles as they rise, although some of them succeed in maintaining their integrity and are still present in the upper part of the water column. Fig. 11 confirms that the LIM approach severely underestimates the amount of topological reconfigurations experienced by large bubbles, as almost no dispersed gas phase is created. The results provided by the other two models appear qualitatively more realistic, with a gradual increase of the volume fraction of the dispersed phase as one gets closer to the upper free surface. This volume fraction seems to be larger with the GLIM approach, especially in the upper half of the water column. However, it must be kept in mind that the criteria by which large gas structures turn into dispersed bubbles differ between the GLIM and LBM approaches; this may be the reason why more entities belong to the dispersed phase in the former at the instant of time selected in Fig. 11.

\subsection{Gas volume fraction}

To obtain a more quantitative insight into the ability of the various models to reproduce the formation of a dispersed gas phase, Fig. 12 displays the horizontal cross-sectional average of the gas volume fraction, $\left\langle\alpha_{g}\right\rangle$, at two different positions within the water column.

At the lower position ( $10 \mathrm{~cm}$ above the neck), the three models yield similar evolutions of the average gas fraction (Fig. 12(a)). This evolution is characterized by a periodic succession of large sharp peaks, each of which corresponds to the crossing of the control volume by a large bubble. This dynamics just reflects the periodic detachment of large bubbles from the neck. The peak amplitude 

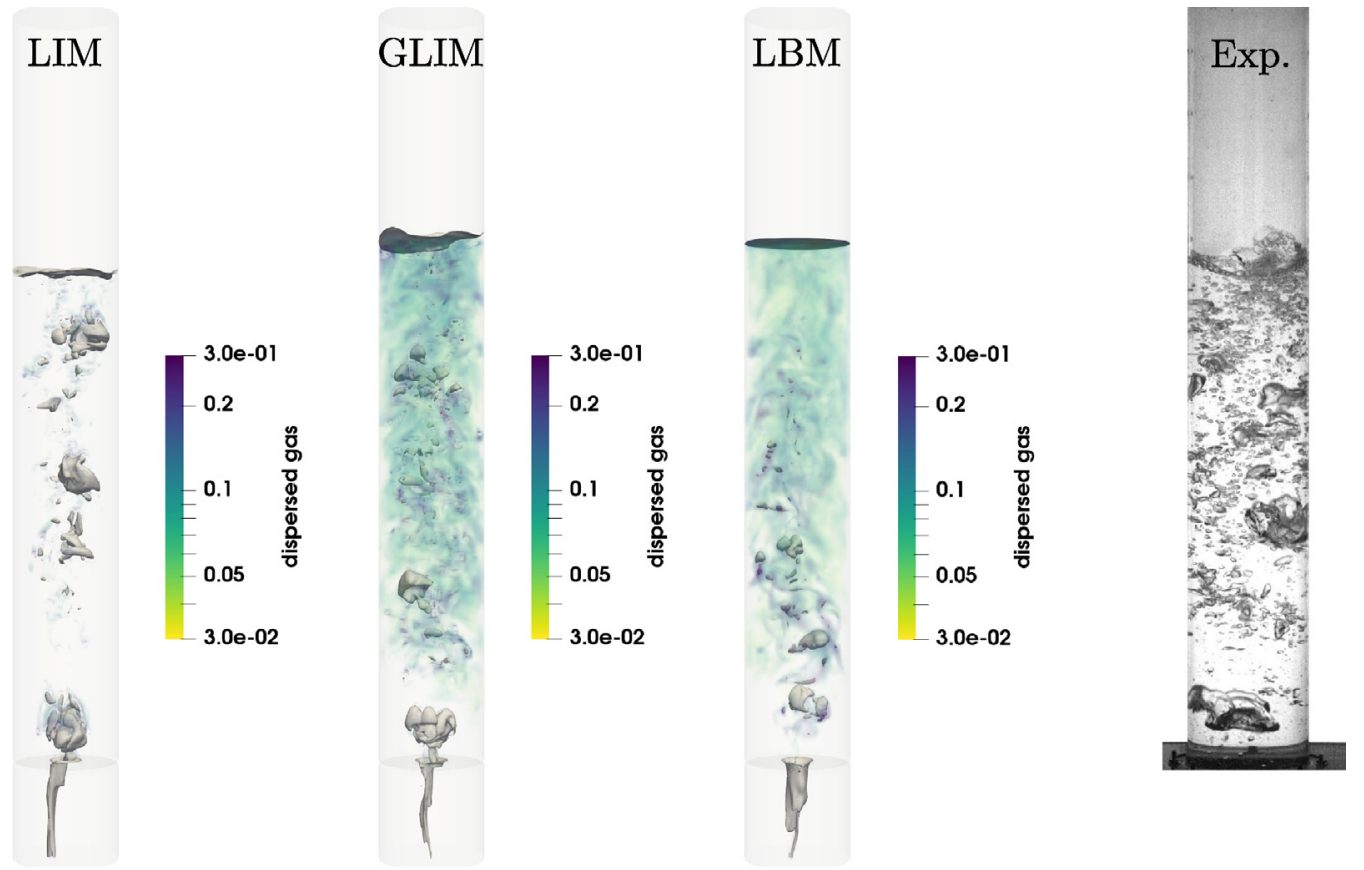

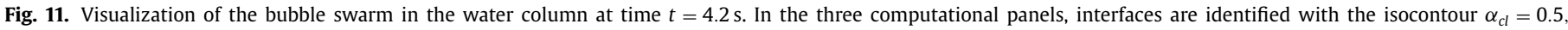

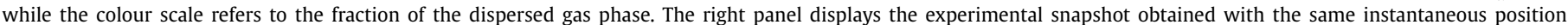
of the upper free surface and an initial condition $z_{0} / L=1$.

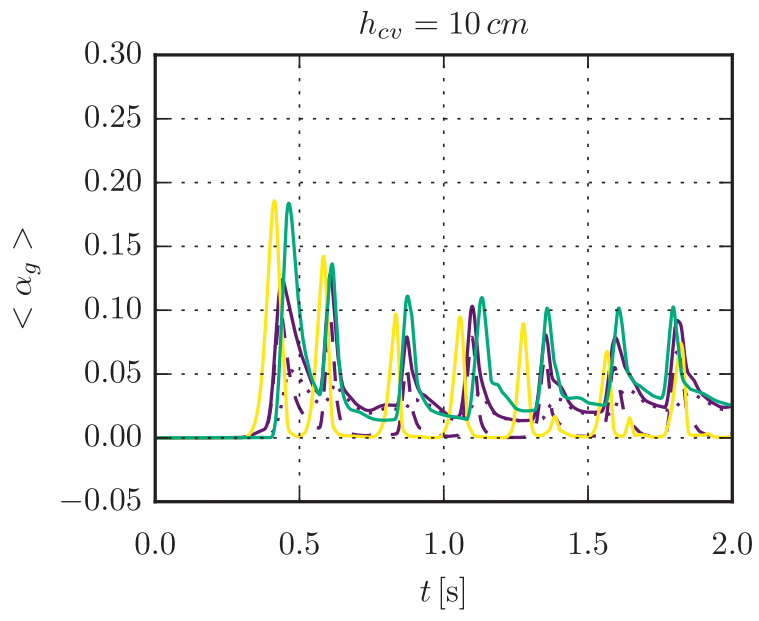

(a)

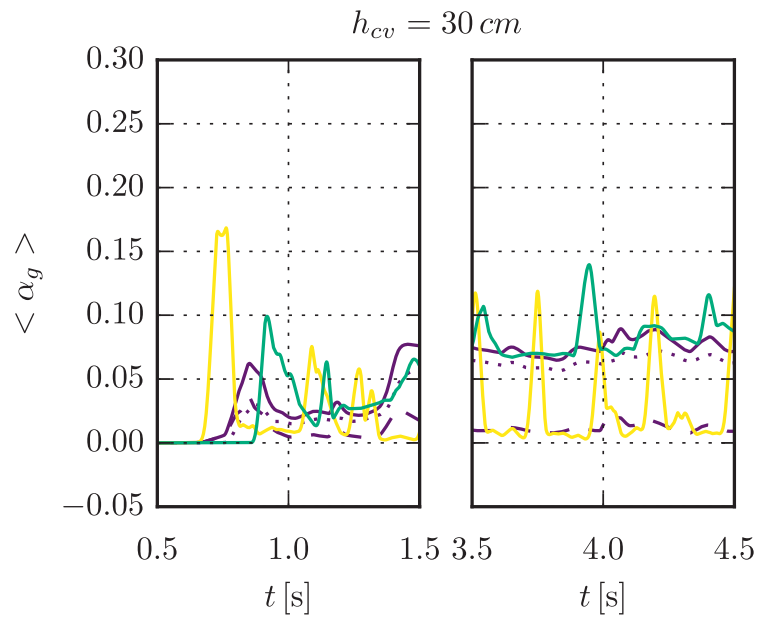

(b)

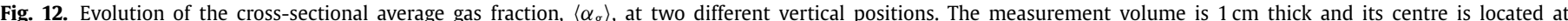

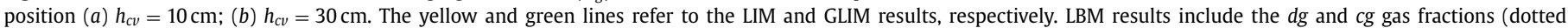

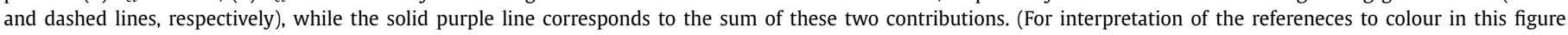
legend, the reader is referred to the web version of this article.)

does not only depend on the size of the large bubbles, but also on their shape and orientation. Indeed, for a given gas volume, a prolate bubble contributes less to the cross-sectional average gas fraction than an oblate one. Similarly, a spherical cap bubble yields a larger contribution than a spherical bubble having the same volume. In between two consecutive peaks, $\left\langle\alpha_{g}\right\rangle$ returns to small values, indicating that the large bubbles are separated by a continuous liquid phase. This is consistent with observations performed at early time after the gate opening. A closer look at Fig. 12(a) reveals that peaks predicted by the GLIM approach are always larger (frequently by a significant percentage) than those obtained with the LBM. The LIM approach also predicts larger peaks during the initial transient (up to $t \approx 1 \mathrm{~s}$ ), before these peaks become smaller than those observed with the LBM. In line with the inability of the LIM approach to 'feed' the dispersed gas phase with initially large bubbles, values of $\left\langle\alpha_{g}\right\rangle$ in between consecutive peaks are virtually zero in the LIM results, whereas they stay in between 2 and $3 \%$ with the other two models.

The evolution of the average gas volume at the higher position $(30 \mathrm{~cm}$ above the neck) is displayed in Fig. 12(b); for the sake of clarity, the short- and long-time evolutions are shown separately. The evolution of $\left\langle\alpha_{g}\right\rangle$ obtained with the LIM approach is similar to that observed at the lower position, with a periodic alternation of large peaks separated by near-zero values, and a magnitude of these peaks that does not vary significantly over time. In line with the previous comments regarding Fig. 11, these observations in- 


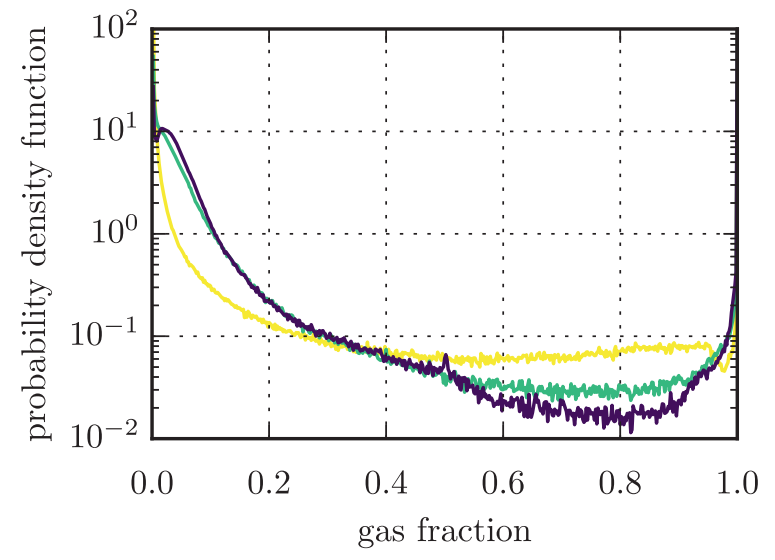

Fig. 13. Pdf of the gas fraction throughout the bottle at time $t=2 \mathrm{~s}$. In the LBM approach, the $c g$ and $d g$ fields have been added to make the comparison with the predictions of the other two models possible. Yellow, green and purple lines refer to the LIM, GLIM and LBM approaches, respectively. (For interpretation of the references to colour in this figure legend, the reader is referred to the web version of this article.)

dicate that the large bubbles generated at the neck roughly keep their size, and presumably their overall shape, unchanged throughout their rise and are not subject to significant fragmentation. The other two models reveal striking differences with respect to LIM predictions. With the GLIM approach, the first large bubble that crosses the test section is detected $0.9 \mathrm{~s}$ after the gate opening, while it is detected $0.15 \mathrm{~s}$ earlier in the LIM simulation. Somewhat later, the average gas fraction increases to reach a value close to $7 \%$ that stays almost constant until the end of the simulation. This increase followed by a 'plateau' value is associated with the generation of a large number of small bubbles in the water column due to the fragmentation of large bubbles, a process that is not considered in the LIM simulation. Results obtained with the LBM approach show that, beyond $t \approx 1.5 \mathrm{~s}$, air is essentially contained in the discrete gas phase (dotted line in Fig. 12), suggesting that the fragmentation process is strong enough to almost turn all large structures that initially belong to the $c g$ phase into small unresolved structures that fill the $d g$ phase. Focusing on the long-term evolution, one can observe that large bubbles are still detected by the GLIM approach whereas they no longer occur in the evolution predicted by the LBM. As experiments also reveal the presence of such large bubbles at this stage, this is an indication that the LBM overestimates the generation of dispersed bubbles compared to the GLIM approach. Quantitative experimental measurements of the local and average gas volume fractions are required to better discriminate the predictions provided by the three models. Nevertheless, the available visualizations already suggest that fragmentation is severely under-predicted in LIM simulations and presumably over-predicted by the LBM approach.

\subsection{Pdf of the gas volume fraction}

The probability density function (pdf) of the gas fraction throughout the entire bottle is plotted in Fig. 13 at time $t=2 \mathrm{~s}$. The gas fraction distribution may be decomposed into three main regions of interest. Regions with gas fractions up to $\approx 0.3$ correspond to those where a dispersed gas phase is present (Taitel et al., 1980). Regions containing gas structures bounded by LI contribute to the central zone of the pdf, around the median iso-value $\alpha_{g}=0.5$. Last, regions with gas fractions $\alpha_{g} \gtrsim 0.6$ correspond to those containing large bubbles and to the top air buffer. Once the flow is established and the first bubbles have reached the top air buffer, the pdf remains almost constant as time proceeds. As the left part of the figure confirms, much less dispersed gas phase is generated with the LIM approach than with the other two models. The pdf obtained with the GLIM and LBM approaches are very similar up to $\alpha_{g} \approx 0.45$. The three models provide significantly different distributions for larger gas fractions. It is no surprise that results obtained with the LIM approach exhibit the highest probability to produce and maintain large bubbles throughout the water column. The large difference observed between the LIM and GLIM predictions for $\alpha_{g} \gtrsim 0.45$ is of special interest, as both models make use of the same technique to detect LI. However, in the LIM approach, gas entities not bounded by a LI still experience the penalization force defined in Eq. (11). In contrast they do not experience added-mass and shear-induced lift forces, the effect of which is known to move bubbles toward the centre of vortical structures (Magnaudet and Eames, 2000; Climent et al., 2007). Conversely, thanks to the switching factor $\gamma$ implemented in the GLIM approach (see Eq. (12)), these forces are properly taken into account in the form given by Eqs. (8) and (9) in that model, provided that the local gas fraction is small enough. What the difference observed in the right half of the two pdf suggests is that the dispersion of small gas bubbles which is made possible by these inertial forces tends to move these bubbles apart from LI, thus lowering the number of flow regions in which the gas fraction maintains large values. The GLIM and LBM approaches are found to yield quite similar pdf, although, compared to LIM results, the depletion of flow regions with large gas fractions is even larger with the LBM. Finally, it may be noticed that a small peak emerges for $\alpha_{g}=0.5$ in the pdf obtained with the LBM approach. This is a direct consequence of the interface sharpening technique (see Section 2.5.1) which tends to concentrate the gas and liquid volume fractions toward the dividing value $\alpha_{g}=\alpha_{l}=0.5$ in LI regions.

\subsection{Pdf of the vertical liquid velocity}

To obtain additional insight into the flow dynamics, we now examine the pdf of the vertical liquid velocity component, say $U_{l}^{z}$, obtained with the three modelling options. Only cells with $\alpha_{l} \geq 0.5$ are considered to compute the pdf. Moreover, to obtain more converged statistics, three velocity fields, each one corresponding to a slightly different instant of time, are used to build the histograms. For instance, the histogram said to correspond to $t=1.3 \mathrm{~s}$ actually involves velocity fields recorded at times $t=1.25,1.30$ and $1.35 \mathrm{~s}$. Fig. 14 displays the evolution of the corresponding pdf at six successive instants of time, up to $t=3.1 \mathrm{~s}$ after the gate opening. Only results obtained with the GLIM approach are reported but the other two models yield similar comments. The corresponding snapshots revealing the instantaneous structure of the bubble swarm are displayed in Fig. 15.

Water being initially at rest, it first flows out of the bottle, until the pressure drop within the top air buffer makes the generation of a first bubble possible. This is why only negative values are involved in the pdf corresponding to $t=0.1 \mathrm{~s}$ in Fig. 14 (purple line); three peaks associated with strong negative flow accelerations may be identified in this pdf, one at each instant of time used to build it. In the second pdf ( $t=0.7 \mathrm{~s}$, dark blue line), the first bubble is rising in the water column, whereas a large part of the liquid is still at rest. This is the origin of the large peak noticed for $U_{l}^{z}=0$ in that pdf. However, the water dragged in the bubble wake has already started rising, resulting in significant probabilities to observe positive velocities up to $\approx 0.7 \mathrm{~m} . \mathrm{s}^{-1}$ in some parts of the bottle. All subsequent pdf (starting with the blue-green line corresponding to $t=1.3 \mathrm{~s}$ after the gate opening) exhibit virtually the same negative branch. This is an indication that the glug-glug dynamics is established after $1.3 \mathrm{~s}$. In contrast, the positive branch goes on evolving significantly from one pdf to the other. This is due to the 


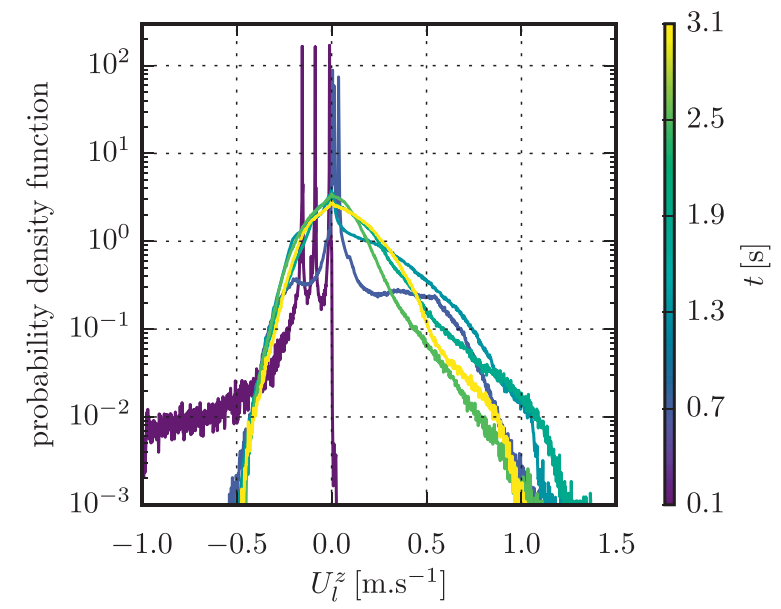

Fig. 14. Successive pdf of the vertical liquid velocity, $U_{l}^{z}$, throughout the water column, from $t=0.1 \mathrm{~s}$ to $t=3.1 \mathrm{~s}$ after the gate opening, as predicted by the GLIM approach. Positive (resp. negative) vertical velocities correspond to liquid inflows (resp. outflows). (For interpretation of the references to colour in this figure legend, the reader is referred to the web version of this article.)

frequent topological changes experienced by the large rising bubbles, which in turn modify in a quite random manner the distribution of upward velocities in the surrounding liquid, especially in wake regions. Note that positive velocities up to $0.5 \mathrm{~m} . \mathrm{s}^{-1}$ are frequently encountered during the whole sequence, which highlights how strong the agitation level throughout the glug-glug process is.

Note also that the pdf discussed throughout this section were built without weighting each sample of the vertical velocity by the radial position at which it is recorded. Hence the flow rate cannot be directly inferred from these pdf. This is why in Fig. 14, pdf corresponding to $t \geq 0.7 \mathrm{~s}$ are positive on average, although the overall flow rate is negative.

Fig. 16 shows the pdf of $U_{l}^{z}$ obtained with all three interfacial momentum exchange models at time $t=3.1 \mathrm{~s}$. The first striking difference observed among the three pdf is the much higher probability to find strong upward velocities, as large as $1 \mathrm{~m} . \mathrm{s}^{-1}$, with the LIM approach. This is again a clear signature of the significantly higher number of large bubbles that coexist within the water column when this approach is employed, as already discussed in Section 5.2. As such bubbles have significantly higher rising speeds than small bubbles, the water they displace forward also rises faster, especially in the wake regions. Examination of the

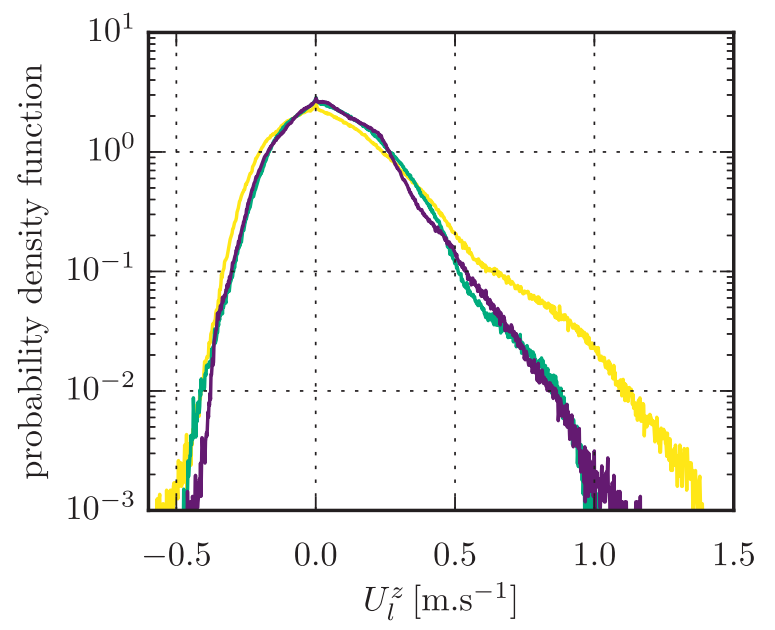

Fig. 16. Pdf of the vertical liquid velocity, $U_{l}^{z}$, obtained with the three different interfacial momentum exchange models at $t=3.1 \mathrm{~s}$. Yellow, green and purple lines refer to the LIM, GLIM and LBM approaches, respectively. (For interpretation of the references to colour in this figure legend, the reader is referred to the web version of this article.)

negative tails of the three pdf, especially in the range $-0.35 \mathrm{~m} . \mathrm{s}^{-1}$ $\leq U_{l}^{z} \leq-0.1 \mathrm{~m} \cdot \mathrm{s}^{-1}$, indicates that events with such large downward velocities also occur significantly more frequently with the LIM approach. This is in agreement with the results of Table 2 in which we already noticed that this approach predicts a somewhat faster emptying process (see the discussion in Section 4.4).

\section{Summary and concluding remarks}

The present paper aims at contributing to the assessment of the capabilities and possible deficiencies of three recently developed interfacial momentum exchange closures available in the EulerEuler NEPTUNE_CFD code, based on detailed comparisons with experimental results obtained in a reference configuration. We selected the test case corresponding to the emptying of a water bottle because, despite its simple geometry, it involves a wide and continuous range of bubble sizes, from large air pockets to tiny bubbles, all of which strongly interact during the emptying process. Thus we expected this two-phase configuration to be relevant for assessing the performances of closure models aimed at provid-

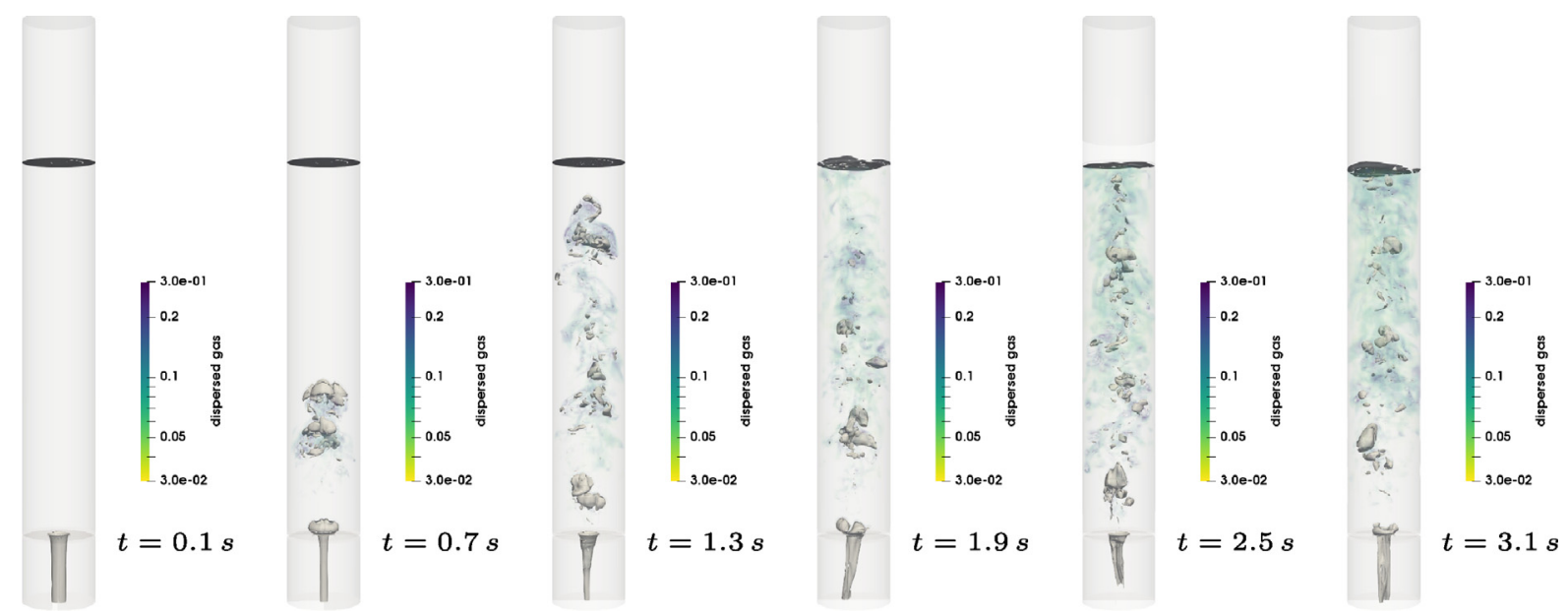

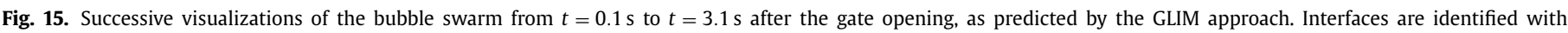
the isocontour $\alpha_{c l}=0.5$, while the colour scale refers to the fraction of the dispersed gas phase. 
ing realistic predictions in complex multi-scale two-phase configurations of industrial relevance. We first detailed these three momentum exchange closures to clarify the assumptions on which they are grounded, especially the nature (physical vs. numerical) of these assumptions.

The comparison of experimental and computational results first confirms that in the selected configuration air compressibility plays an essential role in the emptying dynamics, as it drives the pressure oscillations within the air buffer located on top of the water column, and these oscillations control the bubble formation process at the neck. We found that, provided this compressibility is taken into account, the three models under consideration correctly reproduce the period and magnitude of these pressure oscillations. They also correctly capture the length of the initial transient during which the oscillation amplitude decays exponentially, but significantly overestimate the corresponding damping rate.

Not surprisingly, the distribution of the gas volume fraction within the water column was observed to vary dramatically from one model to the other. A qualitative comparison of the local flow structure predicted by each of them with experimental visualizations allowed us to conclude that the LIM approach grossly underestimates the fragmentation rate of the large bubbles created at the neck. Consequently, this model is unable to reproduce the swarm of small bubbles observed experimentally. The GLIM and LBM approaches, both of which involve criteria by which the "continuous' gas phase may turn into a 'dispersed' phase, do a better job in that respect and are in better qualitative agreement with experiments. We confirmed these trends by examining several aspects of the pdf of the gas volume fraction, showing in particular that this pdf is strongly biased toward large gas fractions when the LIM approach is used. We finally examined the pdf of the vertical velocity in the liquid, the behaviour of which is tightly coupled with that of the gas volume fraction, owing to the entrainment process in wake regions. Tails of these pdf behave similarly in the GLIM and LBM approaches but differ significantly from those obtained in LIM simulations. In particular, as less upward entrainment takes place in the latter, the probability to observe intense downward jetting events is larger, resulting in a slightly larger average flow rate.

In the configuration investigated here and with the cutoff lengths involved in the GLIM and LBM approaches kept unchanged, small air bubbles were found to only slightly affect most of the overall flow characteristics. Nevertheless, compared to the LIM representation, a noticeable reduction of the liquid flow rate was observed with the above two approaches. Influence of the cutoff length selected in these models has still to be examined, as does that of the isothermal condition which must be assessed by running the same computations assuming an isentropic evolution.

The present configuration may be further used to achieve more quantitative comparisons regarding the predictions provided by the various models. To this end, we shall first examine the possible influence of the precise shape of the neck on the emptying dynamics, keeping in mind that for the time being computations were carried out with a cylindrical neck whereas a bevelled geometry is employed in experiments. This will allow us to get rid of the potential influence of this geometrical detail, so as to make sure that any difference left with the experimental results may be attributed to some aspect of the closure models. The next step of our investigation will then focus on experiments. More precisely, we now plan to perform local determinations of the gas volume fraction and bubble size distribution within the water column. Indeed this is a key step to quantitatively discriminate the capabilities and shortcomings of the closure laws discussed in Section 2. This information is also needed to enrich some of them, especially the mass exchange closures (see Eqs. (18) and (19)) aimed at cap- turing the consequences of break-up and coalescence events. This simple configuration, in which multiple topological changes take place, may provide interesting data to improve this complementary and less explored aspect of the closure procedure.

\section{Acknowledgements}

The authors acknowledge the contribution of Dandara Velasco who obtained and gathered the experimental data during her Master thesis at IMFT. This work was granted access to the HPC resources of the CALMIP supercomputing centre under the allocation P17034.

\section{Supplementary material}

Supplementary material associated with this article can be found, in the online version, at doi:10.1016/j.ijmultiphaseflow.2018. 05.002 .

\section{References}

Adoua, R., Legendre, D., Magnaudet, J., 2009. Reversal of the lift force on an oblate bubble in a weakly viscous linear shear flow. J. Fluid Mech. 628, 23-41.

Auton, T.R., 1987. The lift force on a spherical body in a rotational flow. J. Fluid Mech. 183, 199-218.

Auton, T.R., Hunt, J.R., Prud'homme, M., 1988. The force exerted on a body in inviscid unsteady non-uniform rotational flow. J. Fluid Mech. 197, 241-257.

Balachandar, S., Eaton, J.K., 2010. Turbulent dispersed multiphase flow. Annu. Rev. Fluid Mech. 42, 111-133.

Bartosiewicz, Y., Laviéville, J., Seynhaeve, J.M., 2008. A first assessment of the NEPTUNE-CFD code: Instabilities in a stratified flow comparison between the VOF method and a two-field approach. Int. J. Heat Fluid Flow 29, 460-478.

Batchelor, G.K., 1967. An Introduction to Fluid Dynamics. Cambridge University Press.

Biesheuvel, A., Spoelstra, S., 1989. The added mass coefficient of a dispersion of spherical gas bubbles in liquid. Int. J. Multiph. Flow 15, 911-924.

Brackbill, J.U., Kothe, D.B., Zemach, C., 1992. A continuum method for modeling surface tension. J. Comput. Phys. 100, 335-354.

Brocchini, M., Peregrine, D., 2001. The dynamics of strong turbulence at free surfaces. Part 1. Description. J. Fluid Mech. 449, 225-254.

Brocchini, M. Peregrine, D., 2001. The dynamics of strong turbulence at free surfaces. Part 2. Free-surface boundary conditions. J. Fluid Mech. 449, 255-290.

Bunner, B., Tryggvason, G., 2002. Dynamics of homogeneous bubbly flows. Part 1 . Rise velocity and microstructure of the bubbles. J. Fluid Mech. 466, 17-52.

Cano-Lozano, J.C., Martinez-Bazán, C., Magnaudet, J., Tchoufag, J., 2016. Paths and wakes of deformable nearly spheroidal rising bubbles close to the transition to path instability. Phys. Rev. Fluids 1, 053604

Černe, G., Petelin, S., Tiselj, I., 2001. Coupling of the interface tracking and the two-fluid models for the simulation of incompressible two-phase flow. J. Comput. Phys. 171, 776-804.

Clanet, C., Searby, G., 2004. On the glug-glug of ideal bottles. J. Fluid Mech. 510, $145-168$.

Climent, E., Simonnet, M., Magnaudet, J., 2007. Preferential accumulation of bubbles in Couette-Taylor flow patterns. Phys. Fluids 19, 083301

Coste, P., 2013. A large interface model for two-phase CFD. Nuclear Eng. Des. 255 38-50.

Deendarlianto, Höhne, T., Lucas, D., Vallée, C., Montoya Zabala, G.A., 2011. CFD studies on the phenomena around counter-current flow limitations of gas/liquid two-phase flow in a model of a PWR hot leg. Nuclear Eng. Des. 241, 5138-5148.

Denèfle, R., 2013. Modélisation locale diphasique eau-vapeur des écoulements dans les générateurs de vapeur. University of Bordeaux 1, France Ph.D. thesis.

Denèfle, R., Mimouni, S., Caltagirone, J.-P., Vincent, S., 2015. Multifield hybrid approach for two-phase flow modeling - Part 1: adiabatic flows. Comput. Fluids 113, 106-111.

Drew, D.A., Passman, S.L., 1999. Theory of Multicomponent Fluids. Springer.

Fleau, S., 2017. Multifield Approach and Interface Locating Method for Two-Phase Flows in Nuclear Power Plant. Univ. Paris Est Marne-La-Vallée, France Ph.D. thesis.

Fleau, S., Mimouni, S., Mérigoux, N , Vincent, S, 2015. Simulations of two-phase flows with a multifield approach. In: Proc. CHT-15. 6th Int. Symp. Adv. Comput. Heat Transf., 2015, Rutgers Univ., New Brunswick, NJ, USA, pp. 78-96.

Fleau, S., Mimouni, S., Merigoux, N., Vincent, S., 2016. Validation of a multifield approach for the simulations of two-phase flows. Comput. Therm. Sci. 7, 441-457.

Gada, V.H., Tandon, M.P., Elias, J., Vikulov, R., Lo, S., 2017. A large scale interface multi-fluid model for simulating multiphase flows. Appl. Math. Model. 44, 189-204.

Geiger, F., Velten, K., Methner, F.J., 2012. 3D CFD simulation of bottle emptying processes. J. Food Eng. 109, 609-618.

Hansch, S., Lucas, D., Krepper, E., Hohne, T., 2012. A multi-field two-fluid concept for transitions between different scales of interfacial structures. Int. J. Multiphase Flow 47, 171-182. 
Henriques, A., 2006. Prise en compte des grandes interfaces dans un code moyenné eulérien à deux champs de vitesse. Inst. Nat. Polytech. Grenoble, France Ph.D. thesis.

Hirt, C.W., Nichols, B.D., 1981. Volume of fluid (VOF) method for the dynamics of free boundaries. J. Comput. Phys. 39, 201-225.

Höhne, T., Vallée, C., 2010. Experiments and numerical simulations of horizontal two phase flow regimes using an interfacial area density model. J. Comput. Multiphase Flows 2, 131-143.

Ishii, M., 1975. Thermo-Fluid Theory of Two-Phase Flow. Eyrolles.

Ishii, M., Hibiki, T., 2006. Thermo-Fluid Dynamics of Two-Phase Flow. Springer Verlag.

Ishii, M., Zuber, N., 1979. Drag coefficient and relative velocity in bubbly, droplet or particulate flows. AIChE J. 25, 843-855.

Issa, R.I., Bonizzi, M., Barbeau, S., 2006. Improved closure models for gas entrainment and interfacial shear for slug flow modelling in horizontal pipes. Int. J. Multiph. Flow 32, 1287-1293.

Issa, R.I., Kempf, M.H.W., 2003. Simulation of slug flow in horizontal and nearly horizontal pipes with the two-fluid model. Int. J. Multiph. Flow 29, 69-95.

Krepper, E., Lucas, D., Frank, T., Prasser, H.M., Zwart, P.J., 2008. The inhomogeneous MUSIG model for the simulation of polydispersed flows. Nuclear Eng. Des. 238, 1690-1702.

Legendre, D., Magnaudet, J., 1998. The lift force on a spherical bubble in a viscous linear shear flow. J. Fluid Mech. 368, 81-126.

Magnaudet, J., Eames, I., 2000. The motion of high-Reynolds-number bubbles in inhomogeneous flows. Annu. Rev. Fluid Mech. 32, 659-708.

Merigoux, N., Lavieville, J., Mimouni, S., Guingo, M., Baudry, C., 2016. A Generalized Large Interface to dispersed bubbly flow approach to model two-phase flows in nuclear power plant. In: CFD4NRS-6, Cambridge, MA - USA, Paper 11-1, pp. 1-20.

Mimouni, S., Boucker, M., Laviéville, J., Guelfi, A., Bestion, D., 2008. Modelling and computation of cavitation and boiling bubbly flows with the NEPTUNE-CFD code. Nuclear Eng. Des. 238, 680-692.

Mimouni, S., Fleau, S., Vincent, S., 2017. CFD calculations of flow pattern maps and LES of multiphase flows. Nuclear Eng. Des. 321, 118-131.

Mimouni, S., Foissac, A., Lavieville, J., 2011. CFD modelling of wall steam condensation by a two-phase flow approach. Nuclear Eng. Des. 241, 4445-4455.

Mimouni, S., Lamy, J.-S., Lavieville, J., Guieu, S., Martin, M., 2010. Modelling of sprays in containment applications with A CMFD code. Nuclear Eng. Des. 240, 2260-2270.

Mimouni, S., Laviéville, J., Seiler, N., Ruyer, P., 2011. Combined evaluation of second order turbulence model and polydispersion model for two-phase boiling flow and application to fuel assembly analysis. Nuclear Eng. Des. 241, 4523-4536.

Montoya, G., Baglietto, E., Lucas, D., 2015. Implementation and validation of a surface tension model for the multi-scale approach GENTOP. In: Int. Top. Meet. Nucl. React. Therm. Hydraul. 2015, NURETH 2015, 5, pp. 4219-4232.

Olsson, E., Kreiss, G., 2005. A conservative level set method for two phase flow. J. Comput. Phys. 210, 225-246.

Olsson, E., Kreiss, G., Zahedi, S., 2007. A conservative level set method for two phase flow II. J. Comput. Phys. 225, 785-807.
Osher, S., Sethian, J.A., 1988. Fronts propagating with curvature-dependent speed: algorithms based on Hamilton-Jacobi formulations. J. Comput. Phys. 79, 12-49.

Patankar, S.V., Spalding, D.B., 1972. A calculation procedure for heat, mass and momentum transfer in three-dimensional parabolic flows. Int. J. Heat Mass Transf. $15,1787-1806$.

Popinet, S., 2009. An accurate adaptive solver for surface-tension-driven interfacial flows. J. Comput. Phys. 228, 5838-5866.

Porombka, P., Höhne, T., 2015. Drag and turbulence modelling for free surface flows within the two-fluid Euler-Euler framework. Chem. Eng. Sci. 134, 348-359.

Prosperetti, A., Tryggvason, G., 2007. Computational Methods for Multiphase Flow. Cambridge University Press.

Rudman, M., 1997. Volume-tracking methods for interfacial flow calculations. Int. J Numer. Methods Fluids 24, 671-691.

Rudman, M., 1998. A volume-tracking method for incompressible multifluid flows with large density variations. Int. J. Numer. Methods Fluids 28, 357-378.

Sato, Y., Ničeno, B., 2012. A conservative local interface sharpening scheme for the constrained interpolation profile method. Int. J. Numer. Methods Fluids 70, 441-467.

Scardovelli, R., Zaleski, S., 1999. Direct numerical simulation of free-surface and interfacial flow. Annu. Rev. Fluid Mech. 31, 567-603.

Sethian, J., Smereka, P., 2003. Level set methods for fluid interfaces. Annu. Rev. Fluid Mech. 35, 341-372.

Sethian, J.A., 1999. Level set methods and fast marching methods, 2nd ed. Cambridge University Press.

Štrubelj, L., Tiselj, I., Mavko, B., 2009. Simulations of free-surface flows with implementation of surface tension and interface sharpening in the two-fluid model. Int. J. Heat Fluid Flow 30, 741-750.

Taitel, Y., Bornea, D., Dukler, A., 1980. Modeling flow pattern transitions for steady upward gas-liquid flow in vertical tubes. AIChE J. 26, 345-354.

Tomiyama, A., Sakoda, K., Hayashi, K., Sou, A., Shimada, N., Hosokawa, S., 2006 Modeling and hybrid simulation of bubbly flow. Multiph. Sci. Technol. 18, $73-110$

Tomiyama, A., Tamai, H., Zun, I., Hosokawa, S., 2002. Transverse migration of single bubbles in simple shear flows. Chem. Eng. Sci. 57, 1849-1858.

Tryggvason, G., Bunner, B., Esmaeeli, A., Juric, D., Al-Rawahi, N., Tauber, W., Han, J., Nas, S., Jan, Y.-J., 2001. A front-tracking method for the computations of multiphase flow. J. Comput. Phys. 169, 708-759.

Unverdi, S., Tryggvason, G., 1992. A front-tracking method for viscous, incompressible multi-fluid flows. J. Comput. Phys. 100, 25-37.

Vallée, C., Höhne, T., Prasser, H.M., Sühnel, T., 2008. Experimental investigation and CFD simulation of horizontal stratified two-phase flow phenomena. Nucl. Eng. Des. 238, 637-646.

Yan, K., Che, D., 2010. A coupled model for simulation of the gas-liquid two-phase flow with complex flow patterns. Int. J. Multiph. Flow 36, 333-348.

Zuber, N., 1964. On the dispersed two-phase flow in the laminar flow regime. Chem. Eng. Sci. 19, 897-917. 